\title{
Dianthi herba: a comprehensive review of its botany, traditional use, phytochemistry, and pharmacology
}

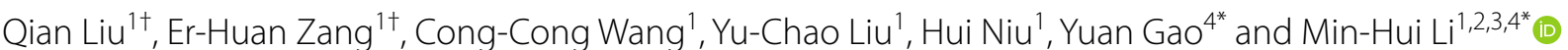

\begin{abstract}
Dianthi herba (called "Qumai" in Chinese) is the dried aerial part of Dianthus superbus L. and Dianthus chinensis L. The species are mainly distributed in the temperate and warm temperate regions in the northern hemisphere, and some regions in Africa and Oceania, as well as South America. However, the distribution pattern of Dianthi herba has not been reviewed. In this review, we summarize the research progress on the botany, traditional use, phytochemistry, pharmacology, toxicology, and clinical applications of Dianthi herba. Approximately 194 chemical compounds have been identified and isolated from Dianthi herba, the most important being triterpenoid saponins, flavonoids, and volatile oil compounds. These compounds possess antiviral, anticancer, antioxidant, and antimicrobial properties, inter alia. Further studies should be carried out on Dianthi herba to elucidate more of its active principles and their mechanisms of action.
\end{abstract}

Keywords: Dianthi herba, Botany, Traditional use, Phytochemistry, Pharmacology, Clinical application

\section{Background}

Dianthi herba is a traditional Chinese medicine (TCM) known as "Qumai." According to the Pharmacopoeia of the People's Republic of China (PRC; 2020), the authentic varieties of Dianthi herba are derived from the dried aerial parts of Dianthus superbus L. and Dianthus chinensis L., belonging to the family Caryophyllaceae, known as "Qumai" and "Shizhu" (Pharmacopoeia Commission of PRC, 2020), respectively. The first record of Dianthi herba is in Shennong Bencaojing (the Classic of Herbal Medicine). Traditionally, it has been used to cure diuresis, invigorate blood circulation, and regulate menstruation $[1,2]$. Owing to its good efficacy, Dianthi herba is

\footnotetext{
*Correspondence: gaoyuan_0524@sina.com; prof_liminhui@yeah.net

${ }^{\dagger}$ Qian Liu and Er-Huan Zang contributed equally to this paper

1 Department of Pharmacy, Baotou Medical College, Baotou 014040, Inner Mongolia, China

${ }^{4}$ Inner Mongolia Hospital of Traditional Chinese Medicine, Hohhot 010020, Inner Mongolia, China

Full list of author information is available at the end of the article
}

often used in combination with other TCMs to treat diseases. For example, Gualou Qumai pills, which contain Dianthi herba, Trichosanthis Fructus, Poria, Dioscoreae rhizoma, and Aconiti Lateralis Radix Praeparata, is a traditional prescription for treating dysuria, edema, and polydipsia [3]. Moreover, Dianthi herba is widely used in Mongolian medicine. Dianthi herba is most frequently used as a variety of "Basaga" in Mongolian medicine compound preparation [4]. Common clinical dosage forms of Dianthi herba include Shenmai-7 decoction, Clove- 8 Wei powder, Zandan-4 decoction, Wu Lingzhi-5 decoction, and Digeda- 4 decoction. Digeda-4 decoction has been included in the Encyclopedia of Mongolian MedicineMongolian Medicine and The Pharmaceutical Standards of the Ministry of Health-Mongolian Medicine Volume. The present review revealed that 194 compounds from $D$. superbus and $D$. chinensis have been reported, and they are mainly classified as saponins, flavonoids, peptides, anthraquinones, phenolic acids, amides, phenylpropanoids, and others. Modern pharmacological studies have original author(s) and the source, provide a link to the Creative Commons licence, and indicate if changes were made. The images or other third party material in this article are included in the article's Creative Commons licence, unless indicated otherwise in a credit line to the material. If material is not included in the article's Creative Commons licence and your intended use is not permitted by statutory regulation or exceeds the permitted use, you will need to obtain permission directly from the copyright holder. To view a copy of this licence, visit http://creativecommons.org/licenses/by/4.0/. The Creative Commons Public Domain Dedication waiver (http://creativeco mmons.org/publicdomain/zero/1.0/) applies to the data made available in this article, unless otherwise stated in a credit line to the data. 
shown that Dianthi herba has antitumor, antioxidant, antiviral, anti-inflammatory, diuretic, uterine excitatory, antimicrobial, and neuroprotective properties $[5,6]$.

In this paper, we used PubMed, ScienceDirect, Web of Science, Springer, Wiley, and the China National Knowledge Infrastructure (CNKI) to collect the relevant literature on Dianthi herba from 1959 to 2021 and reviewed its botany, traditional application, phytochemistry, pharmacological properties, toxicity, and clinical applications (Fig. 1 visually shows the thinking of this paper). We aimed to provide a comprehensive review of Dianthi herba to determine its therapeutic potential and indicate directions for future research that will serve as a basis for the further development and utilization of this resource.

\section{Botany}

The family Caryophyllaceae comprises approximately 2000 plant varieties distributed worldwide. D. superbus is mainly distributed in the temperate and warm temperate regions of the northern hemisphere, particularly in northern Europe, central Europe, Siberia, Kazakhstan, Mongolia (western and northern), Korea, Japan, and China, and in some regions in Africa and Oceania, as well as South America (Fig. 2). D. chinensis inhabits regions similar to those of D. superbus and is mainly distributed in Kazakhstan, Korea, Mongolia, Russia, and Europe (Fig. 3). The species distribution data are from the
Global Biodiversity Information Facility (GBIF)-is an international network and data infrastructure funded by the world's governments and aimed at providing anyone, anywhere, open access to data about all types of life on Earth. GBIF Secretariat currently manages and maintains GBIF.

Dianthus superbus is an herbaceous perennial plant, growing to a height of $50-60 \mathrm{~cm}$ with stems that are clumped and branched at the upper part. The leaf blades are linear-lanceolate, $5-10 \times 0.3-0.5 \mathrm{~cm}$ in size, and the apex is acuminate and connate at the base forming a sheath. There are one to two terminal or axillary flowers with two to three pairs of bracts that are obovate and $0.6-1 \times 0.4-0.5 \mathrm{~cm}$ in size. The apex has a long cusp, while the upper edge of the petal is lobed into a fringed shape. The plants blossom from June to September, and the fruit appears from August to October. This plant is widely cultivated in China where it grows in hilly mountain forests, forest margins, meadows, valleys, and other locations at altitudes of 400-3700 m (Flora Reipublicae Popularis Sinicae, 2019).

Dianthus chinensis is an herbaceous perennial plant that grows to a height of $30-50 \mathrm{~cm}$, and the whole plant is hairless. The stems arise from rhizomes and are sparsely clustered with erect upper branches. The leaf blades are linear-lanceolate and $3-5 \times 0.2-0.4 \mathrm{~cm}$ in size, with an apex that is acuminate and connate at the base forming

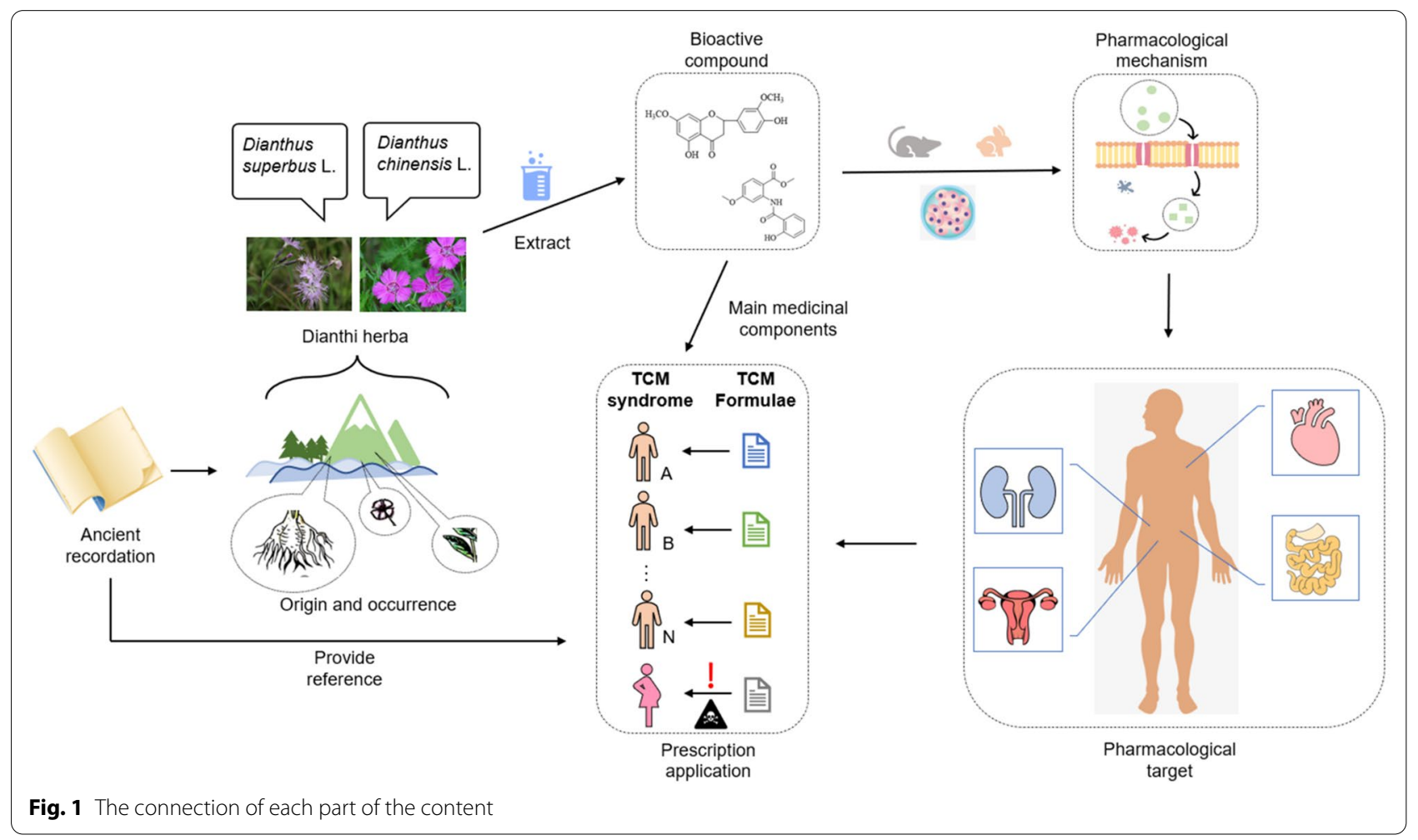




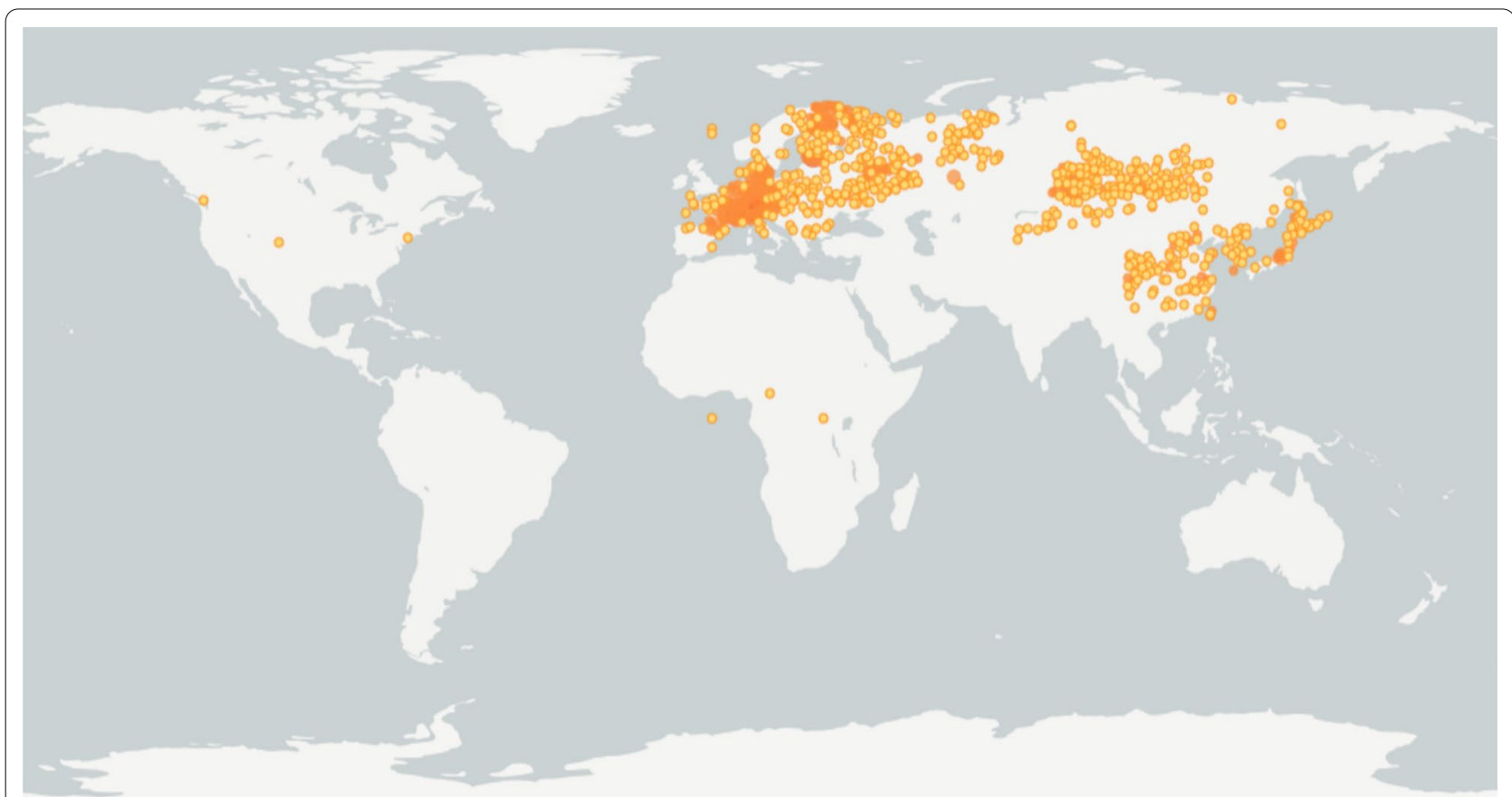

Fig. 2 Distribution map of D. superbus by GBIF ( https://www.gbif.org/). The distribution proportion of D. superbus is 7.3\% in Asia, 92.4\% in Europe, and $0.3 \%$ in North America and South America. More detailed coordinate information shows this in more detail (see Additional file 1)

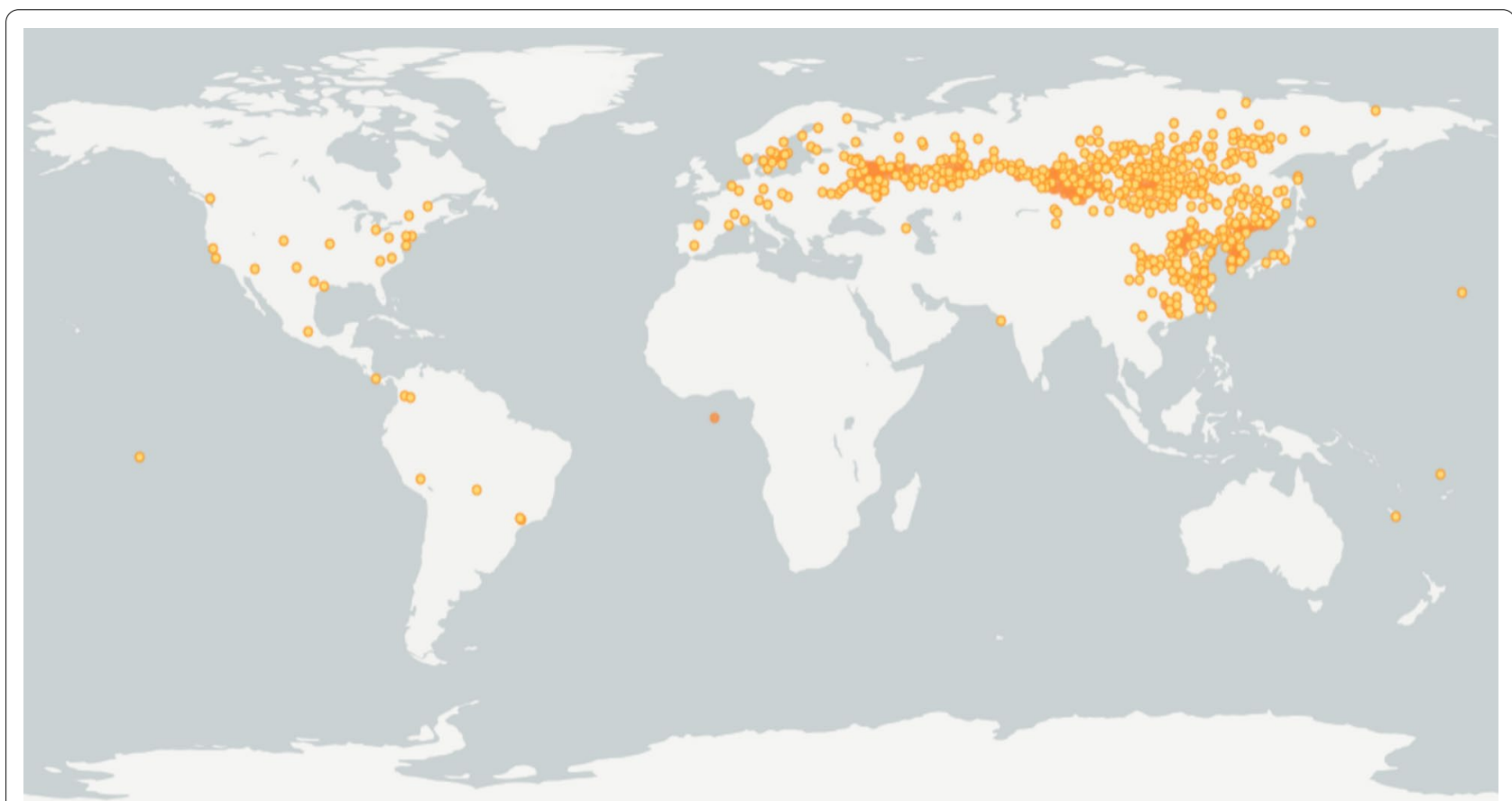

Fig. 3 Distribution map of D. chinensis by GBIF ( https://www.gbif.org/). The distribution proportion of D. chinensis is 35.1\% in Asia, 57.6\% in Europe, and $4.1 \%$ in North America and 3.2\% in South America. More detailed coordinate information shows this in more detail (see Additional file 1) 
a sheath. The flowers are solitary branchlets that are terminal or compound umbels and approximately 1-3 cm long. There are four bracts, while the length of the sepal is half, the apex has a long cusp, and the upper edge of the petal is tooth-shaped. The plant blossoms from May to June, and the fruits appear from July to September. This plant is native to northern China and currently grows in northern and southern regions, grasslands, hillside meadows, and other regions (Flora Reipublicae Popularis Sinicae, 2019). The plant morphologies of D. superbus and $D$. chinensis are shown in Fig. 4.

\section{Traditional uses}

TCMs have been used to treat various diseases, and the long history of use and superior safety profile of TCM formulations have increased the confidence of public in TCMs and its acceptance. Dianthi herba is one of the few commonly used TCMs, and is recorded in ancient Chinese books and Chinese herbal medicine manuals in successive dynasties. It has been documented under different names in several well-known medicinal records, including Shennong Bencaojing (called "Jujumai"), Guang Ya (called "Ziwei"), Ri Hua Zi Ben Cao (called "Shizhu". This is the name of "Shizhu" for the first time.), and $Q i$ Min Yao Shu (called "Dimian", because its seeds can be made into cakes to eat), and in contemporary medicinal records, such as Zhong Hua Ben Cao and The Chinese Dictionary of Herbal Medicine. Qumai is a combination of seeds, stems, and leaves and was first mentioned in Shennong Bencaojing. The textual legend of the plant is shown in Ben Cao Jing Ji Zhu, the plant is described to have a fruit resembling wheat. The plant morphology of a carnation "stem with distinct nodes, leaves opposite, striate, bract subcalyx, petal margins shallowly toothed" has been accurately described in the book of Zhi Wu Ming Shi Tu Kao. Since the Liang Dynasty, Dianthus has been used as a medicinal herb in the name of "Qumai", which has become the main plant source of the TCM "Qumai." The detailed traditional uses are shown in Table 1.

Ancient books have described the characteristics of Dianthi herba. Dianthi herba tastes bitter, and is cold and non-toxic. Dianthi herba is mainly used as a diuretic to help relieve the pain during urination. Its clinical uses include the treatment of urinary tract infections, red and astringent urination, dysmenorrhea, red eye, eye swelling and pain, esophageal cancer, and rectal cancer. Dianthi herba has a wide range of medicinal uses and is mostly prescribed clinically (Table 2). Furthermore, Dianthi herba is used as medicine by ethnic minorities in China. Dianthi herba is recorded in Zhonghua Materia Medica-Mongolian Medicine Volume; it is indicated for blood fever, blood tingling, liver fever, and other diseases

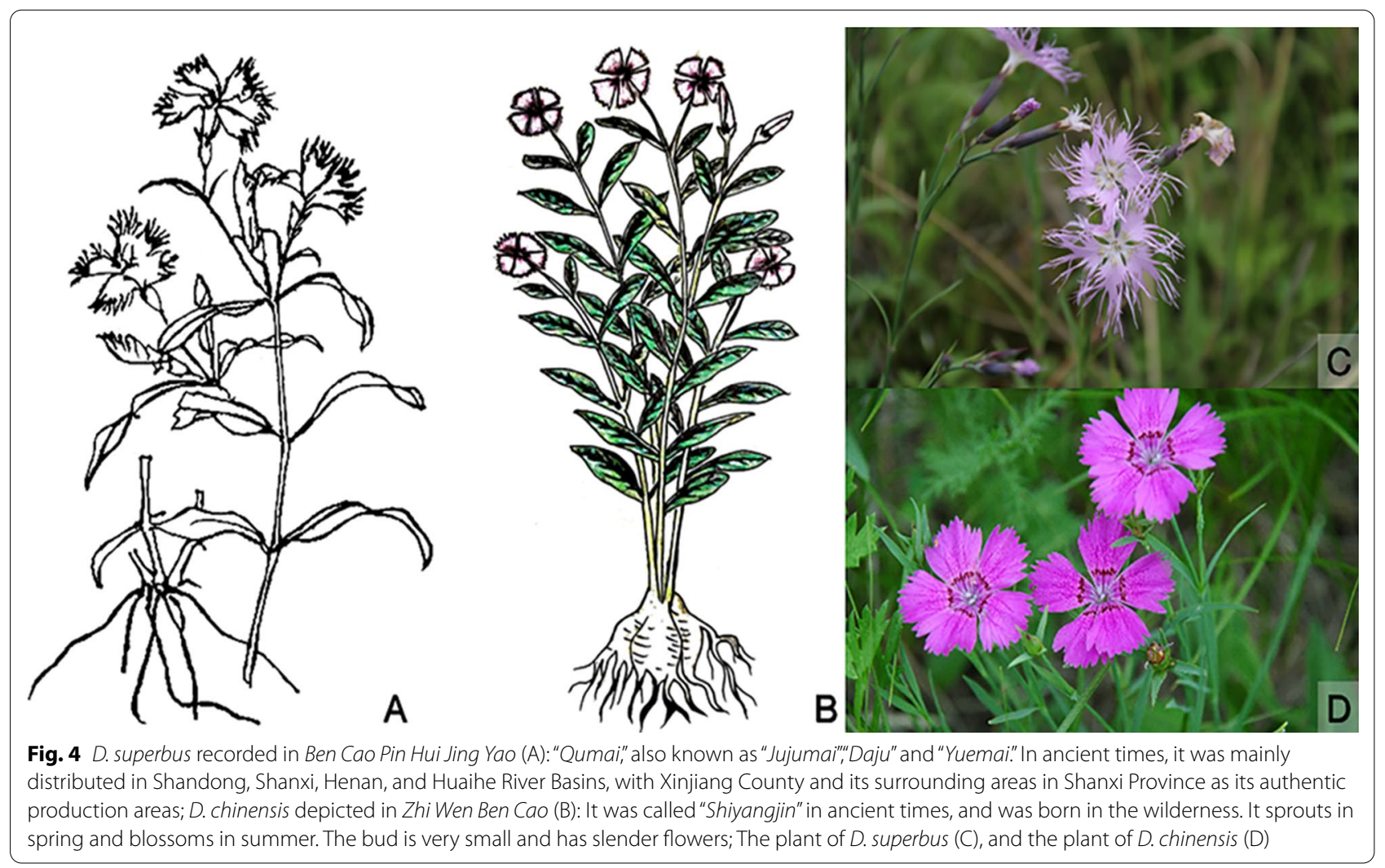


Table 1 The detailed traditional uses of Dianthus superbus L. as medicine

\begin{tabular}{|c|c|c|c|}
\hline Species & Traditional uses & Traditional Chinese medicine with the same traditional uses & Refs \\
\hline \multirow[t]{9}{*}{ Dianthus superbus $\mathrm{L}$} & Hemorrhoid and fistula & $\begin{array}{l}\text { Nepeta cataria L.; Lonicerae Japonicae Flos; Sophorae Flos; Gentiana macrophylla } \\
\text { Pall.; Sophorae Fructus; Coptidis Rhizoma; Rehmanniae Radix; Notoginseng Radix } \\
\text { Et Rhizoma; Astragalus mongholicus Bunge; Codonopsis Radix; Mume Fructus }\end{array}$ & $\begin{array}{l}\text { Ri Hua Zi Ben } \\
\text { Cao, } 1578 \text { C.E. } \\
{[107]}\end{array}$ \\
\hline & Cysts & $\begin{array}{l}\text { Astragalus mongholicus Bunge; Codonopsis Radix; Salviae Miltiorrhizae Radix Et } \\
\text { Rhizoma; Atractylodis Macrocephalae } \\
\text { Rhizoma; Sparganii Rhizoma; Curcuma phaeocaulis Valeton; Sophorae Flavescentis } \\
\text { Radix }\end{array}$ & {$[88,108-110]$} \\
\hline & Anti-early pregnancy & Pinelliae Rhizoma; Carthami Flos; Arnebiae Radix & {$[38,111-113]$} \\
\hline & Diabetic nephropathy & $\begin{array}{l}\text { Dioscoreae Rhizoma; Cornus officinalis Sieb. et Zucc.; Alismatis Rhizoma; Persicae } \\
\text { Semen }\end{array}$ & {$[89,114]$} \\
\hline & Chronic prostatitis & $\begin{array}{l}\text { Angelicae Sinensis Radix; Ginseng Radix Et Rhizoma; Epimedium brevicornu } \\
\text { Maxim.; Tripterygium wilfordii Hook. f.; Ginseng Radix Et Rhizoma Rubra }\end{array}$ & {$[90,92,115,116]$} \\
\hline & Pelvic inflammatory disease & $\begin{array}{l}\text { Salviae Miltiorrhizae Radix Et Rhizoma; Paeoniae Radix Rubra; Violae Herba; } \\
\text { Taraxaci Herba }\end{array}$ & {$[91,117]$} \\
\hline & Vulnerary and Amenorrhea & $\begin{array}{l}\text { Angelicae Sinensis Radix; Cyperi Rhizoma; Ligusticum chuanxiong; Codonopsis } \\
\text { Radix }\end{array}$ & {$[93,105,118]$} \\
\hline & Gonorrhea & Smilax glabra Roxb.; Kochiae Fructus; Portulaca oleracea L.; Hedyotis diffusa Willd & {$[94,106,119]$} \\
\hline & Antiphlogistic and Diuretic & Phyllanthus urinaria L.; Lygodll Spora; Emilia sonchifolia (L.) DC & {$[71,78,95,120]$} \\
\hline
\end{tabular}

such as phase stroke and puerperal fever [4]. In China, Dianthi herba is sold commercially based on the external features of the dried aerial part. Currently, most medicinal products of Dianthi herba sold in the market are sourced from $D$. chinensis and $D$. superbus, but there are also products containing adulterants, such as $D$. chinensis var. versicolor and D. superbus var. longicalycinus [7-9].

\section{Phytochemistry}

Approximately 194 compounds including saponins, flavonoids, volatile oils, and cyclic peptides have been identified in D. superbus and D. chinensis. These compounds and their corresponding structures are shown in Figs. 5, $6,7,8,9,10,11,12$ and Table 3.

\section{Saponins}

Saponins are one of the main chemical constituents of $D$. superbus and D. chinensis. Oleanane-type triterpenoid saponins have been identified as characteristic constituents of these plants [10]. Nineteen triterpenoid and three steroid saponins have been isolated and identified in $D$. superbus and $D$. chinensis. The triterpenoid saponins 12-diene-23,28-dioic acid 28-O- $\beta$-D-glucopyranoside (1), 3-O- $\beta$-D-glucopyranosyl olean-11,13(18)-diene23,28 -dioic acid 28-O- $\beta$-D-glucopyranoside (2), $3-O-\beta$-D-glucopyranosyl olean-28-O-[ $\beta$-D-O- $((3 \mathrm{~S})-3-$ hydroxyl-3-methlglutaryl)-glucopyranosyl- $(1 \rightarrow 6)]-\beta$-Dglucopyranoside (3), 3-O- $\beta$-D-glucopyranosyl gypsogenic acid 28-O-[ $\beta$-D-glucopyranosyl $(1 \rightarrow 3)]$ $[\beta-\mathrm{D}-6-((3 \mathrm{~S})-\mathrm{h}$ yd r ox yl - $3-2 \mathrm{~m}$ e th lgluta r yl $)-$ glucopyranosyl- $(1 \rightarrow 6)]-\beta$-D-glucopyranoside $(4)$, 3-O- $\alpha-\mathrm{L}-$ arabinopyranosyl-3b,16a-dihydroxyolean-12-en-23,28-dioicacid
28-O-[ $\beta$-D-glucopyranosyl- $(1 \rightarrow 6)]-\beta$-D-glucopyranoside (5), and asiatic acid (6) have been isolated from the dried aerial parts of $D$. superbus [11-14]. Four triterpenoid saponins, dianchinenoside A-H (7-14) were isolated from the dried aerial parts of $D$. chinensis $[15,16]$. The compound, 3-O- $\alpha$-L-arabinopyranosyl hederagenin $28-O-\beta$ D-glucopyranosyl $(1 \rightarrow 6)$ - $\beta$-D-glucopyranoside $(15)$ was isolated from the dried aerial parts of $D$. chinensis [17]. The triterpenoid saponins madecassic acid (16), sterculin A (17), (24R)-cycloart-25-ene-3 $\beta, 24$-diol (18), and (24S)cycloart-25-ene-3 $\beta, 24$-diol (19) were isolated from the dried aerial parts of $D$. superbus $[18,19]$. Steroid saponins including $\beta$-spinasterol (20), stigmast-7-en-3 $\beta$-ol (21), $\beta$-sitosterol glucoside (22), Hainanenside (23), 3-O- $\beta$-Dglucopyranosyl olean-11, 13 (18)-diene-23, 28-dioic acid (24) and Quillaic acid (25) have been isolated from the dried aerial parts of D. superbus [20-22] (Fig. 5).

\section{Flavonoids}

Flavonoids are an important class of natural organic compounds with a basic 2-phenyl-chromone structure; they are widely distributed in the plant kingdom. Most natural flavonoids exist in the form of glycosides, which differ in composition according to the type, quantity, linkage position, and connection mode of the sugar. A few flavonoids and flavonoid glycosides have been reported in D. superbus and D. chinensis.

To date, the following 20 flavonoids have been isolated from these plants, including quercetin-3-O-glucoside (26), quercetin-7-O-glucoside (27), isoorientin (28), orientin (29), 5-hydroxy 7,3',4'-trimethoxyflavanone (30), 5,3'-dihydroxy-7,4'-dimethoxyflavanone 


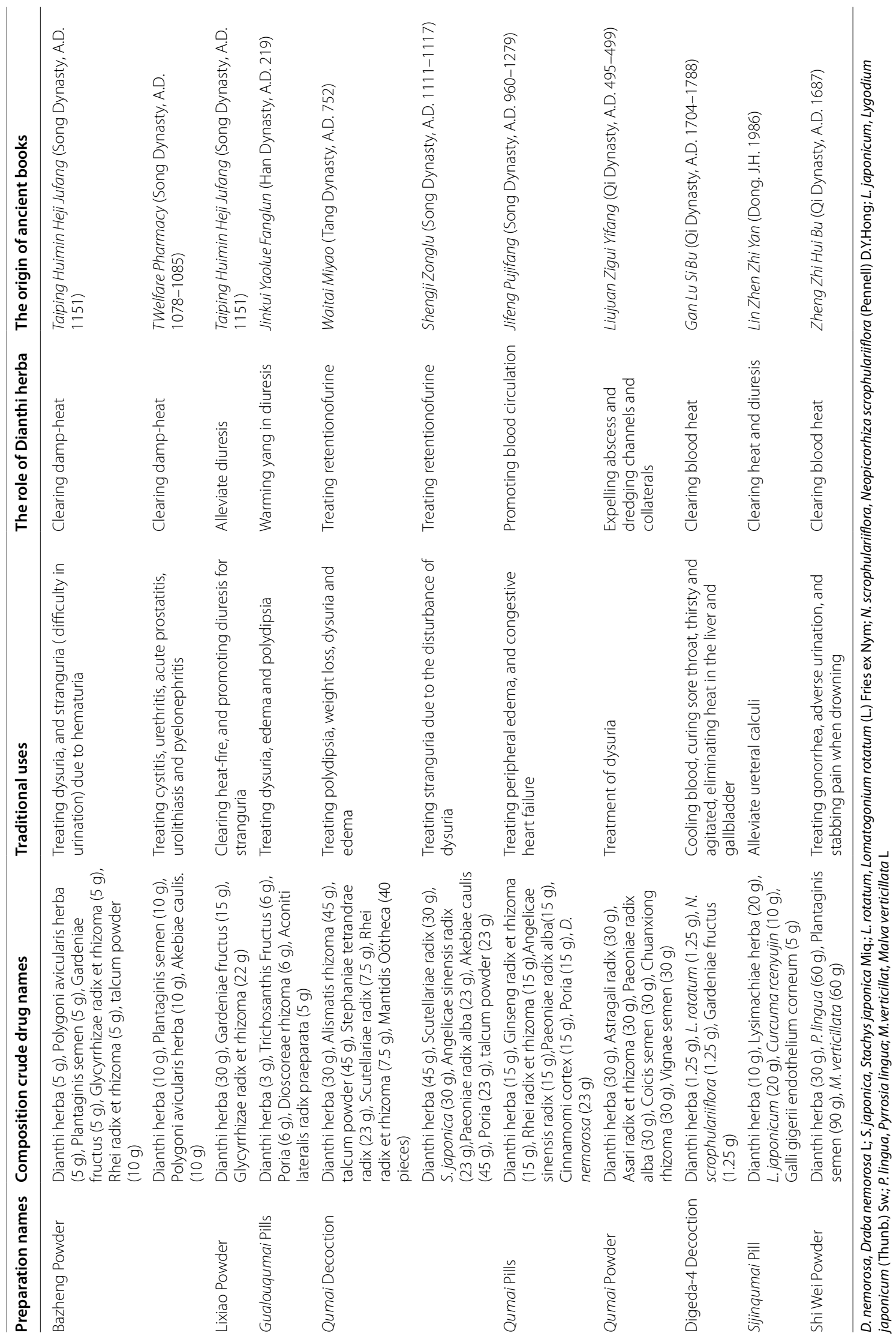




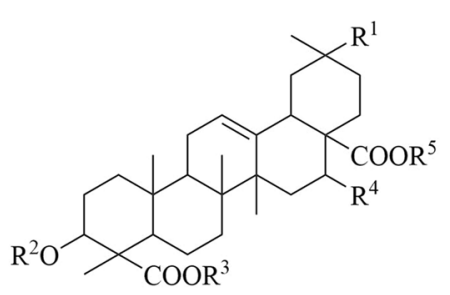

3. $\mathrm{R}^{1}=\mathrm{CH}_{3} \quad \mathrm{R}^{2}=\mathrm{Glu} \quad \mathrm{R}^{3}=\mathrm{H}$

4. $\mathrm{R}^{1}=\mathrm{CH}_{3}$

$\mathrm{R}^{2}=\mathrm{Glu}$

$\mathrm{R}^{3}=\mathrm{H}$

$\mathrm{R}^{3}=\mathrm{H}$

$\mathrm{R}^{4}=\mathrm{H}$

$5 \cdot \mathrm{R}^{1}=\mathrm{CH}_{3} \quad \mathrm{R}^{2}=\mathrm{Glu}$

$\mathrm{R}^{3}=\mathrm{H}$

$7 . \mathrm{R}^{1}=\mathrm{CH}_{3}$

$\mathrm{R}^{2}=$ Ara

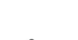

$8 \cdot \mathrm{R}^{\mathrm{l}}=\mathrm{CH}_{3} \quad \mathrm{R}^{2}=\mathrm{Xyl}$

$\mathrm{R}^{3}=\mathrm{H}$

$\mathrm{R}^{3}=\mathrm{Glu}$

9. $\mathrm{R}^{1}=\mathrm{CH}_{3} \quad \mathrm{R}^{2}=\mathrm{H}$

10. $\mathrm{R}^{1}=\mathrm{CH}_{3}$

$\mathrm{R}^{2}=\mathrm{H}$

$\mathrm{R}^{3}=\mathrm{H}$

$\mathrm{R}^{4}=\mathrm{H}$

11. $\mathrm{R}^{1}=\mathrm{CH}_{3} \quad \mathrm{R}^{2}=\mathrm{H}$

$\mathrm{R}^{3}=\overbrace{\mathrm{H}}^{(\mathrm{R})} \mathrm{CH}_{\mathrm{OH}}$

$\mathrm{R}^{4}=\mathrm{OH}$

$\mathrm{R}^{4}=\mathrm{OH}$

$\mathrm{R}^{4}=\mathrm{OH}$

$\mathrm{R}^{4}=\mathrm{OH}$

$\mathrm{R}^{4}=\mathrm{OH}$

$\mathrm{R}^{4}=\mathrm{H}$

$\mathrm{R}^{3}=\overbrace{\mathrm{H}}^{(\mathrm{S})} \mathrm{CH}_{\mathrm{OH}}$

$\mathrm{R}^{4}=\mathrm{H}$

$\mathrm{R}^{3}=\overbrace{\mathrm{H}}^{(\mathrm{R})} \mathrm{CH}_{\mathrm{OH}}$

$\mathrm{R}^{4}=\mathrm{H}$

$\mathrm{R}^{3}=\overbrace{\mathrm{H}^{*}}^{(\mathrm{S})} \mathrm{CH}_{\mathrm{OH}}$

$\mathrm{R}^{4}=\mathrm{H}$

$\mathrm{R}^{3}=\mathrm{H}$

$\mathrm{R}^{4}=\mathrm{OH}$

$\mathrm{R}^{5}=\mathrm{Glu}$

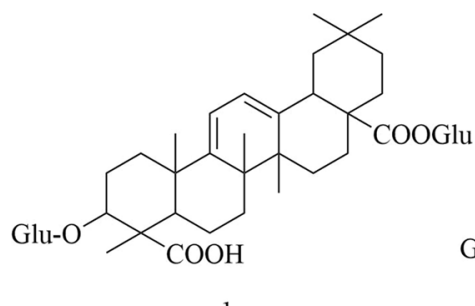

1

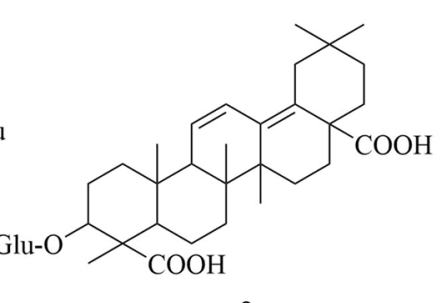

2

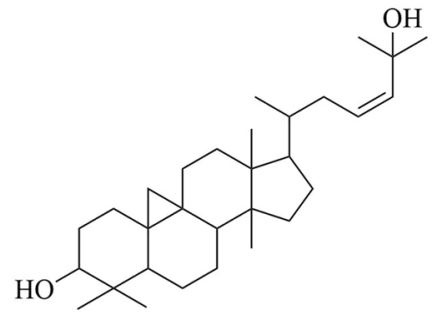

17

Fig. 5 Chemical structures of saponins

(31), 5,4'-dihydroxy-7,3'-dimethoxyflavanone (32), isoorientin-2'-O-glucoside (33), chrysoeriol-7-Oglucoside (34), isovitexin-2"-O-glucoside (35), diosmetin-7-O(2", $6^{\prime \prime}$-di-O- $\alpha$-L-rhamnopyranosyl)-
$\beta$-D-glucopyranoside (36), quercetin-3-O-rutinoside (37), isorhamnetin-3-O-glucoside (38), quercetin-3-O-rhamnoside 7-O-rhamnoside (39), kaempferol-3-O-glucoside-glucoside 7-O-rhamnoside (40), quercetin-3-O-rhamnoside-glucoside 7-O-rhamnoside 
<smiles>C=C(C)[C@H](O)CCC1CCC2(C)C(C)CCC3(C)CC24CCC(O)C(C)(C)C4CCC13</smiles>

18<smiles>CCC(CCC(C)C1CCC2C3=CCC4CC(O)CCC4(C)C3CCC21C)C(C)C</smiles>

21<smiles>[R]C1CCC2(C(=O)O)CCC3(C)C(=CCC4C5(C)CC(O)C(O)C(C)(CO)C5C([R])CC43C)C2C1[R]</smiles>

$6 . \mathrm{R}^{1}=\mathrm{CH}_{3} \quad \mathrm{R}^{2}=\mathrm{CH}_{3} \quad \mathrm{R}^{3}=\mathrm{H}$

16. $\mathrm{R}^{1}=\mathrm{CH}_{3} \quad \mathrm{R}^{2}=\mathrm{CH}_{3} \quad \mathrm{R}^{3}=\mathrm{OH}$<smiles>C=C(C)[C@H](O)CCC(C)C1CCC2(C)C3CCC4C(C)(C)C(O)CCC4(C)C3CCC12C</smiles>

19<smiles>CCC(CCC(C)C1CCC2C3CC=C4CC(OC)CCC4(C)C3CCC12C)C(C)C</smiles>

22<smiles>[R20]C(=O)C12CCC(C)(C)CC1C1=CCC3C([R])([R])C([R2])CCC3(C)C([R])CC12C</smiles>

15. $\mathrm{R}^{1}=\mathrm{Ara} \quad \mathrm{R}^{2}=\mathrm{CHOH} \quad \mathrm{R}^{3}=\mathrm{CH}_{3} \quad \mathrm{R}^{4}=\mathrm{CH}_{3} \quad \mathrm{R}^{5}=\mathrm{Glu}-\mathrm{Glu}$

25. $\mathrm{R}^{1}=\mathrm{H} \quad \mathrm{R}^{2}=\mathrm{CH}_{3} \quad \mathrm{R}^{3}=\mathrm{COH} \quad \mathrm{R}^{4}=\mathrm{OH} \quad \mathrm{R}^{5}=\mathrm{H}$

Fig. 5 continued

(41), luteolin (42), 3,5,7-trihydroxy-3', 5'-dimethoxylflavone (43), kaempferol (44), quercetrin (45), Tricin (46), Diosmetin (47) and 5-Hydroxy-7,3,4'-trimethoxydihydroflavone (48) [18, 20, 22-31] (Fig. 6).

\section{Peptides}

Cyclic peptides are a class of peptides with a variety of applications and have been widely studied over the past decades [32]. Cyclic peptides were first isolated from $D$. superbus in 1998, and they have gradually become a hotspot in chemical research [33]. Ten peptides namely dianthin A-H (49-56), 4-methoxydianthramide B (60), and tyrocidine B (65) were obtained from $D$. superbus $[30,33-35]$. The seven peptides in D. chinensis are dianthin I (57), pseudostellarin A (58), heterophyllin J (59), cyclo-(L-N-methyl Glu-L-Nmethyl Glu (61), cyclo-(L-methyl Glu-ester-L-methyl Glu ester (62), cyclo-(L-methyl Glu-ester-L-Glu) (63), and cyclo-(L-Glu-L-Glu) (64) [36, 37] (Fig. 7).

\section{Anthraquinones}

Three anthraquinones, emodin (66), physcion (67), emodin-8-O- $\beta$-D-glucopyranoside (68), Physcion-8-O- $\beta$ D-glucoside (69), melrubiellinA (70) and melrubiellinB (71) have been isolated from $D$. superbus and D. chinensis [19-21, 25, 38-40] (Fig. 8).

\section{Phenolic acids}

A total of 15 phenolic acids were isolated. Including $p$-Hydroxybenzoic acid (72), 3-Hydroxy-4-methoxybenzoic acid (73), Methyl 3,4-dihydroxybenzoate (74), Methyl 3-(3,4-dihydroxyphenyl) propionate(75), 4-hydroxy3-methoxy-pentyl ester benzenepropanoic acid (76), Vanillic acid (77), 4-Hydroxy-benzeneacetic acid (78), Hydroferulic acid (79), Methyl hydroferulate (80), (E)-methyl-4-hydroxy4-(8a-methyl-3-oxodecahydronaphthalen-4a-yl) (81), Trans-p-coumaric acid (82), Catechol (83), Salicylic acid (84), $\beta$-hydroxypropiovanillone (85) and 4-Methoxyphenylacetic acid (86) [19-21, 26, 31, 38, 41, 42] (Fig. 9). 
<smiles>[R]c1cc(-c2oc3c([R])c([R])c([R])c(O)c3c(=O)c2[R])cc([R])c1[R]</smiles>

$$
\begin{aligned}
& \text { 26. } \mathrm{R}^{1}=\mathrm{H} \quad \mathrm{R}^{2}=\mathrm{OH} \quad \mathrm{R}^{3}=\mathrm{H} \quad \mathrm{R}^{4}=\mathrm{OH} \quad \mathrm{R}^{5}=\mathrm{OH} \quad \mathrm{R}^{6}=\mathrm{OGlu} \\
& \text { 27. } \mathrm{R}^{1}=\mathrm{H} \quad \mathrm{R}^{2}=\mathrm{OGlu} \quad \mathrm{R}^{3}=\mathrm{H} \quad \mathrm{R}^{4}=\mathrm{OH} \quad \mathrm{R}^{5}=\mathrm{OH} \quad \mathrm{R}^{6}=\mathrm{OGlu} \\
& \text { 28. } \mathrm{R}^{1}=\mathrm{H} \quad \mathrm{R}^{2}=\mathrm{OH} \quad \mathrm{R}^{3}=\mathrm{Glu} \quad \mathrm{R}^{4}=\mathrm{OH} \quad \mathrm{R}^{5}=\mathrm{OH} \quad \mathrm{R}^{6}=\mathrm{H} \\
& \text { 29. } \mathrm{R}^{1}=\mathrm{Glu} \quad \mathrm{R}^{2}=\mathrm{OH} \quad \mathrm{R}^{3}=\mathrm{H} \quad \mathrm{R}^{4}=\mathrm{OH} \quad \mathrm{R}^{5}=\mathrm{OH} \quad \mathrm{R}^{6}=\mathrm{H} \\
& \text { 33. } \mathrm{R}^{1}=\mathrm{OH} \quad \mathrm{R}^{2}=\mathrm{OGlu} \quad \mathrm{R}^{3}=\mathrm{Glu} \quad \mathrm{R}^{4}=\mathrm{OH} \quad \mathrm{R}^{5}=\mathrm{OH} \quad \mathrm{R}^{6}=\mathrm{H} \\
& \text { 34. } \mathrm{R}^{1}=\mathrm{OH} \quad \mathrm{R}^{2}=\mathrm{H} \quad \mathrm{R}^{3}=\mathrm{Glu} \quad \mathrm{R}^{4}=\mathrm{OCH}_{3} \quad \mathrm{R}^{5}=\mathrm{OH} \quad \mathrm{R}^{6}=\mathrm{H} \\
& \text { 35. } \mathrm{R}^{1}=\mathrm{H} \quad \mathrm{R}^{2}=\mathrm{OH} \quad \mathrm{R}^{3}=\text { Glu-Glu } \quad \mathrm{R}^{4}=\mathrm{H} \quad \mathrm{R}^{5}=\mathrm{OH} \quad \mathrm{R}^{6}=\mathrm{H} \\
& \text { 36. } \mathrm{R}^{1}=\mathrm{H} \quad \mathrm{R}^{2}=\mathrm{OGlu}^{\text {Rha }} \mathrm{R}^{3}=\mathrm{H} \quad \mathrm{R}^{4}=\mathrm{OCH}_{3} \quad \mathrm{R}^{5}=\mathrm{OH} \quad \mathrm{R}^{6}=\mathrm{H} \\
& \text { 37. } \mathrm{R}^{1}=\mathrm{H} \quad \mathrm{R}^{2}=\mathrm{OH} \quad \mathrm{R}^{3}=\mathrm{H} \quad \mathrm{R}^{4}=\mathrm{OH} \quad \mathrm{R}^{5}=\mathrm{OH} \quad \mathrm{R}^{6}=\text { OGlu-Rha } \\
& \text { 38. } \mathrm{R}^{1}=\mathrm{H} \quad \mathrm{R}^{2}=\mathrm{OH} \quad \mathrm{R}^{3}=\mathrm{H} \quad \mathrm{R}^{4}=\mathrm{OH} \quad \mathrm{R}^{5}=\mathrm{OCH}_{3} \quad \mathrm{R}^{6}=\mathrm{OGlu} \\
& \text { 39. } \mathrm{R}^{1}=\mathrm{H} \quad \mathrm{R}^{2}=\mathrm{OH} \quad \mathrm{R}^{3}=\mathrm{H} \quad \mathrm{R}^{4}=\mathrm{OH} \quad \mathrm{R}^{5}=\mathrm{OH} \quad \mathrm{R}^{6}=\text { ORha-Rha } \\
& \text { 40. } \mathrm{R}^{1}=\mathrm{H} \quad \mathrm{R}^{2}=\mathrm{ORha} \quad \mathrm{R}^{3}=\mathrm{H} \quad \mathrm{R}^{4}=\mathrm{OH} \quad \mathrm{R}^{5}=\mathrm{H} \quad \mathrm{R}^{6}=\text { OGlu-Glu } \\
& \text { 41. } \mathrm{R}^{1}=\mathrm{H} \quad \mathrm{R}^{2}=\mathrm{ORha} \quad \mathrm{R}^{3}=\mathrm{H} \quad \mathrm{R}^{4}=\mathrm{OH} \quad \mathrm{R}^{5}=\mathrm{OH} \quad \mathrm{R}^{6}=\text { ORha-Glu } \\
& 42 . \mathrm{R}^{1}=\mathrm{H} \quad \mathrm{R}^{2}=\mathrm{OH} \quad \mathrm{R}^{3}=\mathrm{H} \quad \mathrm{R}^{4}=\mathrm{OH} \quad \mathrm{R}^{5}=\mathrm{H} \quad \mathrm{R}^{6}=\mathrm{H} \\
& \text { 43. } \mathrm{R}^{1}=\mathrm{H} \quad \mathrm{R}^{2}=\mathrm{OH} \quad \mathrm{R}^{3}=\mathrm{H} \quad \mathrm{R}^{4}=\mathrm{H} \quad \mathrm{R}^{5}=\mathrm{OCH}_{3} \quad \mathrm{R}^{6}=\mathrm{OH} \quad \mathrm{R}^{7}=\mathrm{OCH}_{3} \\
& \text { 44. } \mathrm{R}^{1}=\mathrm{H} \quad \mathrm{R}^{2}=\mathrm{OH} \quad \mathrm{R}^{3}=\mathrm{H} \quad \mathrm{R}^{4}=\mathrm{OH} \quad \mathrm{R}^{5}=\mathrm{H} \quad \mathrm{R}^{6}=\mathrm{OH} \\
& \text { 45. } \mathrm{R}^{1}=\mathrm{H} \quad \mathrm{R}^{2}=\mathrm{OH} \quad \mathrm{R}^{3}=\mathrm{H} \quad \mathrm{R}^{4}=\mathrm{OH} \quad \mathrm{R}^{5}=\mathrm{OH} \quad \mathrm{R}^{6}=\mathrm{OH} \\
& \text { 46. } \mathrm{R}^{1}=\mathrm{H} \quad \mathrm{R}^{2}=\mathrm{OH} \quad \mathrm{R}^{3}=\mathrm{H} \quad \mathrm{R}^{4}=\mathrm{OH} \quad \mathrm{R}^{5}=\mathrm{O} \quad \mathrm{R}^{6}=\mathrm{H} \quad \mathrm{R}^{7}=\mathrm{O} \\
& \text { 47. } \mathrm{R}^{1}=\mathrm{H} \quad \mathrm{R}^{2}=\mathrm{OH} \quad \mathrm{R}^{3}=\mathrm{H} \quad \mathrm{R}^{4}=\mathrm{O} \quad \mathrm{R}^{5}=\mathrm{H} \quad \mathrm{R}^{6}=\mathrm{H} \quad \mathrm{R}^{7}=\mathrm{OH}
\end{aligned}
$$

$$
\begin{array}{llllll}
30 . \mathrm{R}^{1}=\mathrm{H} & \mathrm{R}^{2}=\mathrm{OCH}_{3} & \mathrm{R}^{3}=\mathrm{H} & \mathrm{R}^{4}=\mathrm{H} & \mathrm{R}^{5}=\mathrm{OCH}_{3} & \mathrm{R}^{6}=\mathrm{OCH}_{3} \\
31 . \mathrm{R}^{1}=\mathrm{H} & \mathrm{R}^{2}=\mathrm{OCH}_{3} & \mathrm{R}^{3}=\mathrm{H} & \mathrm{R}^{4}=\mathrm{H} & \mathrm{R}^{5}=\mathrm{OCH}_{3} & \mathrm{R}^{6}=\mathrm{OH} \\
32 . \mathrm{R}^{1}=\mathrm{H} & \mathrm{R}^{2}=\mathrm{OCH}_{3} & \mathrm{R}^{3}=\mathrm{H} & \mathrm{R}^{4}=\mathrm{H} & \mathrm{R}^{5}=\mathrm{OH} & \mathrm{R}^{6}=\mathrm{OCH}_{3} \\
48 . \mathrm{R}^{1}=\mathrm{H} & \mathrm{R}^{2}=\mathrm{O} & \mathrm{R}^{3}=\mathrm{H} & \mathrm{R}^{4}=\mathrm{O} & \mathrm{R}^{5}=\mathrm{O} & \mathrm{R}^{6}=\mathrm{H}
\end{array}
$$

Fig. 6 Chemical structures of flavonoids 

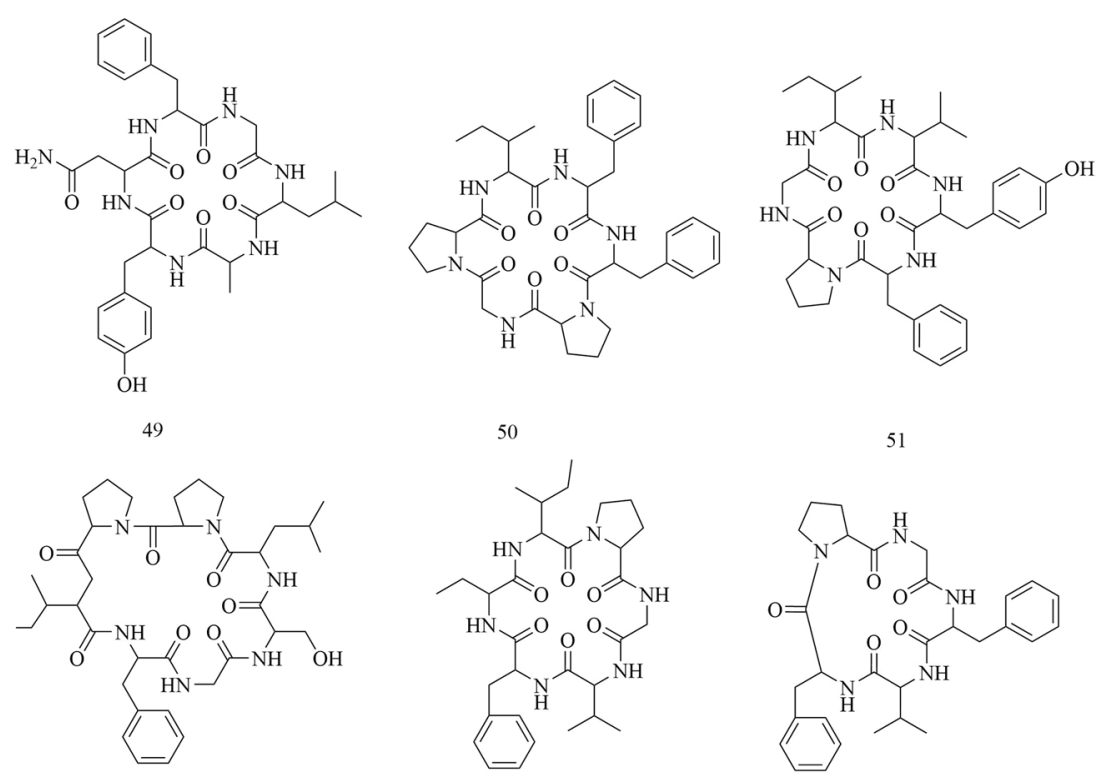

51

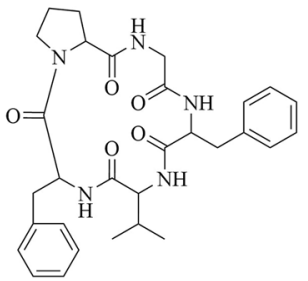

52

53

54
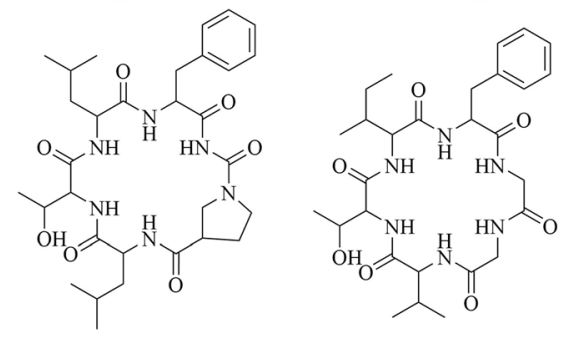

55

56

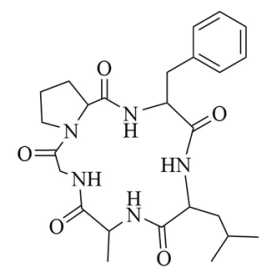

58

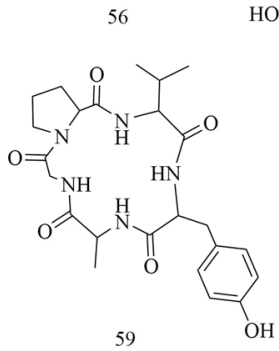<smiles>CNC(CC(N)=O)C(=O)NC(C)CCC(N)=O</smiles>

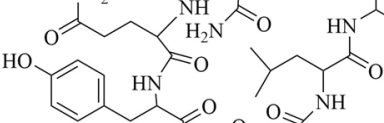<smiles>CC(CNCCc1ccccc1)Cc1c[nH]c2ccccc12</smiles><smiles>CNC(C(=O)NC(CCCN)C(C)=O)C(C)C</smiles>

65

59

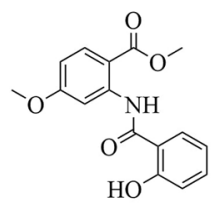

60<smiles>[R20]C(=O)CCC1C(=O)[Y17]([H])=C(CCC(=O)O)C(N([Y7])[Y])C1=O</smiles>

61. $\mathrm{R}^{1}=\mathrm{H}$

$\mathrm{R}^{2}=\mathrm{CH}_{3}$

$\mathrm{R}^{3}=\mathrm{H}$

62. $\mathrm{R}^{1}=\mathrm{CH}_{3}$

$\mathrm{R}^{2}=\mathrm{H}$

$\mathrm{R}^{3}=\mathrm{CH}_{3}$

$\mathrm{R}^{4}=\mathrm{CH}_{3}$

63. $\mathrm{R}^{1}=\mathrm{CH}_{3}$

$\mathrm{R}^{2}=\mathrm{H}$

$\mathrm{R}^{3}=\mathrm{CH}_{3}$

64. $\mathrm{R}^{1}=\mathrm{H}$

$\mathrm{R}^{2}=\mathrm{H}$

$\mathrm{R}^{3}=\mathrm{H}$

$\mathrm{R}^{4}=\mathrm{H}$

Fig. 7 Chemical structures of peptides 


$$
\begin{aligned}
& \text { (c) } \\
& \text { 66. } \mathrm{R}^{1}=\mathrm{H} \\
& \mathrm{R}^{2}=\mathrm{H} \\
& \text { 67. } \mathrm{R}^{1}=\mathrm{H} \\
& \mathrm{R}^{2}=\mathrm{CH}_{3} \\
& \text { 68. } \mathrm{R}^{\mathrm{l}}=\mathrm{Glu} \\
& \mathrm{R}^{2}=\mathrm{H} \\
& \text { 69. } \mathrm{R}^{\mathrm{l}}=\mathrm{Glu} \\
& \mathrm{R}^{2}=\mathrm{CH}_{3}
\end{aligned}
$$

70. $\mathrm{R}=\mathrm{H}$

71. $\mathrm{R}=\mathrm{OH}$

Fig. 8 Chemical structures of anthraquinones<smiles>O=C(O)c1ccc(O)cc1</smiles>

72<smiles>COc1ccc(C(=O)O)cc1O</smiles>

73<smiles>COC(=O)c1ccc(O)c(O)c1</smiles>

74<smiles>COc1cc(C(=O)O)ccc1O</smiles>

77<smiles>COC(=O)CCc1ccc(O)c(OC)c1</smiles>

81<smiles>COc1cc(C(=O)CCO)ccc1O</smiles>

85<smiles>COC(=O)CCc1ccc(O)c(O)c1</smiles>

75

$\mathrm{HO}$<smiles>O=C(O)Cc1ccccc1</smiles>

78<smiles>O=C(O)/C=C/c1ccc(O)cc1</smiles>

82<smiles>COc1ccc(CC(=O)O)cc1</smiles>

86

Fig. 9 Chemical structures of phenolic acids

\section{Amides}

Amides are also found in D. superbus and D. chinensis. Both Patriscabratine (87), N-p-coumarylanthranilic acid (88), Methoxydianthramide S (89), 2-[(2,4-Dihydroxybenzoyl) amino]-4-methoxy-benzoic acid (90), Aurantiamide (91) and Aurantiamide acetate (92) are reported compounds [19, 21, 26, 31] (Fig. 10).

\section{Phenylpropanoids}

Phenylpropanol is a kind of phenolic substance that exists naturally. The special phenylpropa-noid compounds found in D. superbus and D. chinensis are
4-Methoxy-benzeneacetic acid (93), Scopoletin (94), 6-Methoxy-hydroxycoumarin (95), epipinoresinol (96), Pinoresinol (97), Prinsepiol (98), Medioresinol (99), Syringaresinol (100) and Dehydrodiconiferyl alcohol (101) [21, 26, 31, 42] (Fig. 11).

\section{Others}

In addition to the compounds mentioned above, a variety of volatile oils and other compounds, including aromatic and aliphatic compounds, have also been isolated and identified. The compound hydroxydihydrobovolide (102) has been isolated from the dried aerial parts of $D$. 
<smiles>CC(=O)NC(COC(=O)C(Cc1ccccc1)NC(=O)c1ccccc1)Cc1ccccc1</smiles>

87<smiles>COc1ccc(C(=O)O)c(NC(=O)c2ccc(O)cc2O)c1</smiles>

90<smiles>O=C(O)c1ccccc1NC/C=C/c1ccc(O)cc1</smiles>

88

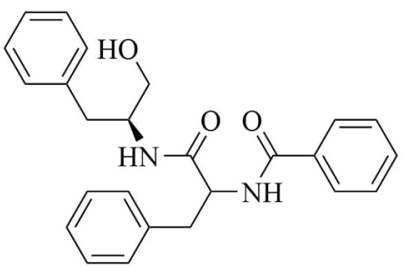

91<smiles>COc1ccc(C(=O)O)c(NC(=O)c2ccccc2O)c1</smiles>

89

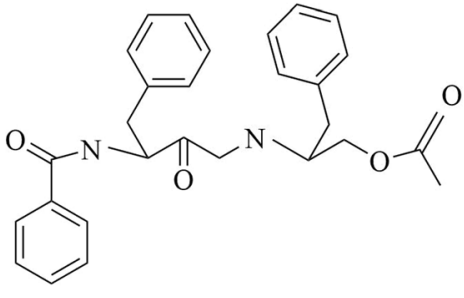

92

Fig. 10 Chemical structures of amides

superbus and extracted and comprehensively analyzed in large amounts from the volatile oils of D. superbus using gas chromatography-mass spectrometry (GC-MS) [18, 19]: cis-3-hexen-1-ol (103), phenylethane (104), $n$-hexanol (105), sorbic acid (106), benzaldehyde (107), $n$-heptanol (108), 1-octen-3-ol (109), 6-methyl-5-hepten-2-one (110), 2-methyl-2-hepten-6-ol (111), phenylacetaldehyde (112), $p$-tolualdehyde (113), n-octanol (114), (E,E)-3,5-octadien-2-one (115), linalool (116), 1-acetyl-2-methylcyclopentene (117), n-nonanal (118), $n$-nonanol (119), decanal (120), n-decanol (121), $\alpha$-terpineol (122), $\alpha$-cyclocitral (123), cis-chrysanthenyl acetate (124), $(E)$-anethole (125), $\alpha$-damascenone (126), tetrahydrogeranyl acetone (127), geranyl acetone (128), 2,6,10-trimethyltetradecane (129), $\beta$-ionone (130), 3,7,11-trimethy 1-1-dodecanol (131), 2-methylhexadecan-1-ol (132), 6,10,14-trimethyl-2-pentadecanone (133), farnesyl acetone (134), palmitic acid (135), and cis-phytol (136). These components account for $73.45 \%$ of the total volatile oils in D. superbus. The results showed that the main components of the essential oils are 6,10,14-trimethyl-2-pentadecanone (28.39\%), cis-phytol (6.80\%), geranyl acetone (4.65\%), n-hexanol (4.32\%), and farnesyl acetone (3.01\%) [43]. (E)-4-methoxycinnamic acid (137), 3-methoxy-4-hydroxyphenylethanol (138), loliolide (139), and 1-monopalmitin (140) were isolated from the dried aerial parts of $D$. superbus $[26,38,42]$. The compounds (141-179) were identified by GC-MS in the petroleum ether extract from $D$. superbus [44]. The monosaccharide L-Dianose (180) was identified by ${ }^{1} \mathrm{H}-\mathrm{NMR}$ (nuclear magnetic resonance) and ${ }^{13} \mathrm{C}$-NMR data from the aerial parts of $D$. chinensis [24]. Other compounds including Dehydrololiolide (181), Dehydrovomifoliol
(182), Succinic acid (183), Parthenin (184), Vomifoliol (185), Zhebeiresinol (186), Lariciresinol (187), ixerol B (188), Cucumegastigmanes I (189), (6z, 9z)-heptadecadiene (190), 3, 6, 9-nonadecatriene (191), 5-hydroxyl3,4-dimethy-5-pentyl-2(5H)-furanone (192), 1-glycerol palmitate (193) and 3-(3,'4'-Dihydroxyphenyl)lactic acid sodium salt (194) were also found in D. superbus [20, 21, 31] (Fig. 12).

\section{Pharmacology}

Dianthi herba has a long history of medicinal use and has been reported to have various pharmacological activities, including antitumor [46], antioxidant [47], antiviral [48], anti-inflammatory $[49,50]$, diuretic [51], uterine excitatory [52], antimicrobial [53], and neuroprotective activity [42]. These activities are summarized in Table 4.

\section{Antitumor activity}

Studies have shown that the triterpenoids, cyclic peptides, flavonoids, and other components present in Dianthus have antitumor activity $[54,55]$. Zhang et al. used the 3-(4,5-dimethylthiazol-2-yl)-2,5-diphenyltetrazolium bromide (MTT) assay to identify and study the antitumor active ingredients in Dianthus. The active ingredient asiatic acid was obtained from the n-butanol site. It inhibited the proliferation of HepG2, SSMC-7721, SKHEP-1, and Bel-7402 cells, with a half-maximal inhibitory concentration $\left(\mathrm{IC}_{50}\right)$ of $55.4,48.9,55.3$, and $58.8 \mu \mathrm{g} /$ $\mathrm{kg}$, respectively [14].

A study investigated the apoptotic effects of the ethanol extract of $D$. chinensis (EEDC) in the human hepatocellular carcinoma cells HepG2 and found that treatment with 50, 100, 200, and $400 \mu \mathrm{g} / \mathrm{mL}$ EEDC 
<smiles>COc1ccc(CC(C)=O)cc1</smiles>

93<smiles>COc1cc(C2OC[C@@H]3C(c4ccc(O)c(O)c4)OC[C@H]23)ccc1O</smiles>

96<smiles>COc1cc(C2OC[C@]3(O)C(c4ccc(O)c(OC)c4)OC[C@]23O)ccc1O</smiles>

98<smiles>COc1cc(C2OC[C@@H]3C(c4cc(OC)c(O)c(OC)c4)OC[C@@H]23)cc(OC)c1O</smiles>

100<smiles>COc1cc2ccc(=O)oc2cc1O</smiles>

94<smiles>COc1ccc2oc(=O)c(O)cc2c1</smiles>

95<smiles>COc1cc(C2OC[C@H]3COC[C@H]23)ccc1O</smiles>

97<smiles>COc1cc(C2OCC3C(c4cc(OC)c(O)c(OC)c4)OC[C@@H]23)ccc1O</smiles>

99<smiles>COc1cc(C2Oc3c(OC)cc(/C=C/CO)cc3C2CO)ccc1O</smiles>

101

Fig. 11 Chemical structures of phenylpropanoids

for 6,12 , and $48 \mathrm{~h}$ induced time-dependent apoptosis. This induction was associated with the condensation of chromatin, activation of caspases, and cleavage of poly (ADP-ribose) polymerase protein. Moreover, apoptosis induced by $D$. chinensis was attenuated by a caspase inhibitor, indicating an important role of caspases in the effects of the methanol extract of $D$. chinensis (MEDC). Furthermore, D. chinensis did not alter the expression of B-cell lymphoma 2 (Bcl-2)-associated $\mathrm{X}$ protein (Bax) in HepG2 cells, but it selectively downregulated the expression of $\mathrm{Bcl}-2$ and $\mathrm{B}$-cell lymphomaextra-large $(\mathrm{Bcl}-\mathrm{xl})$, resulting in an increase in the Bax:Bcl-2 and Bax:Bcl-xl ratios. These results support a mechanism in which $D$. chinensis induces apoptosis through the mitochondrial pathway and caspase activation in HepG2 cells [56].

The apoptotic activities and molecular mechanisms of action of MEDC were evaluated in human oral cancer cells using the 3-(4,5-dimethylthiazol-2-yl)-5-(3carboxymethoxyphenyl)-2-(4-sulfophenyl)-2H-tetrazolium (MTS) assay, 4',6-diamidino-2-phenylindole (DAPI) staining, immunostaining, western blotting, and reversetranscription polymerase chain reaction (RT-PCR). Sp1 was significantly over-expressed in oral tumor tissues than in normal oral mucosa. The downregulation of Sp1 inhibited the growth of SCC-15 and YD-15 oral cancer cells, whereas $D$. chinensis inhibited cell growth and induced apoptosis in both cell lines by decreasing the 
<smiles>CCCCCC1(O)OC(=O)C(C)=C1C</smiles>

102<smiles>CC/C=C\CCO</smiles>

103<smiles>CCc1ccccc1</smiles>

104<smiles>CCCCCCO</smiles>

105<smiles>C/C=C/C=C/C(=O)O</smiles>

106<smiles>CC(=O)CCC=C(C)C</smiles>

110<smiles>CC(C)=CCCC(C)O</smiles>

111<smiles>O=Cc1ccccc1</smiles>

107<smiles>CCCCCCCO</smiles>

108<smiles>C=CC(O)CCCCC</smiles>

109<smiles>O=CCc1ccccc1</smiles>

112<smiles>Cc1ccc(C=O)cc1</smiles>

113<smiles>C=CC(C)(O)CCC=C(C)C</smiles><smiles>CCCCCCCCC=O</smiles><smiles>CCCCCCCCCO</smiles><smiles>CCCCCCCCCC=O</smiles>

118

119<smiles>CC1=CCC(C(C)(C)O)CC1</smiles>

122

120<smiles>CC1=C(C=O)C(C)(C)CCC1</smiles>

123<smiles>CC(=O)OC12C3CC4C(=C(C)C31)C42C</smiles>

124<smiles>CC(=O)CCC=C(C)CCC=C(C)C</smiles>

128<smiles>C/C=C/c1ccc(OC)cc1</smiles>

125<smiles>CC=CC(=O)C1=C(C)C=CCC1(C)C</smiles>

126<smiles>CC(=O)CCCC(C)CCCC(C)C</smiles>

127<smiles>CC(=O)/C=C/C1C(C)=CCCC1(C)C</smiles>

129

Fig. 12 Chemical structures of others 
<smiles>CC(C)CCCC(C)CCCC(C)CCO</smiles>

131<smiles>CC(=O)CCCC(C)CCCC(C)CCCC(C)C</smiles>

133<smiles>CCCCCCCCCCCCCCCC(=O)O</smiles>

135<smiles>COc1ccc(/C=C/C(=O)O)cc1</smiles>

137<smiles>CCCCCCCCCCCCCCCC(=O)OCC(O)CO</smiles>

140<smiles>CCCCCCCCCCCCCCC(C)CO</smiles>

132<smiles>CC(=O)CC/C=C(\C)CC/C=C(\C)CCC=C(C)C</smiles>

134<smiles>C/C(=C/CO)CCCC(C)CCCC(C)CCCC(C)C</smiles>

136<smiles>CC1(C)CC(O)CC2(C)OC(=O)C=C12</smiles>

139<smiles>CCC(C)CC(C)C</smiles>

141<smiles>COC(=O)CCCCCCCCCCC(C)C</smiles>

142

143

144<smiles>CCC(C)CCCCCCCCCCCC(=O)OC</smiles>

145<smiles>C=CC(C)(O)CCCC(C)CCCC(C)CCCC(C)C</smiles>

146<smiles>COC(=O)CCCCCCCCCCCCCCC(C)C</smiles>

147<smiles>Cc1ccc(C=O)c(C)c1</smiles>

148<smiles>CCC(C)CC(C)CC(C)C</smiles>

149<smiles>CCCCCCCCC(C)C</smiles>

150<smiles>CC(C)CCCCC(C)CCCC(C)C</smiles>

151<smiles>CC(C)(C)c1cccc(C(C)(C)C)c1O</smiles>

152<smiles>COC(=O)CCc1cc(C(C)(C)C)c(O)c(C(C)(C)C)c1</smiles>

153

Fig. 12 continued 
<smiles>CCCCCCCCCCCCCC</smiles>

159

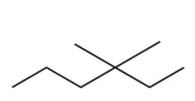

163<smiles>C/C=C/C=C/CCCC</smiles>

155

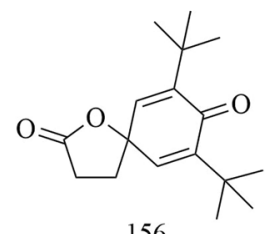

156

154<smiles>CC(=O)OCCCCCCCCCCC(C)C=C(C)C</smiles>

157

158<smiles>CC(=O)/C=C/C1(O)C(C)=CC(=O)CC1(C)C</smiles>

160<smiles>O=C(OCCCCCCCOC(=O)c1ccccc1)c1ccccc1</smiles>

161<smiles>CCCCCCCC(C)CCC=O</smiles>

162<smiles>CCCCCCCCCCCCCCCCCC(C)CCC</smiles>

167

168<smiles>CC/C=C\CCCCCC=O</smiles>

169<smiles>CCCCCC(CC)CO</smiles>

164<smiles>CCCCCO</smiles>

165<smiles>CCCCCc1ccco1</smiles>

166<smiles>COCCCCCCCCCOC</smiles>

171<smiles>c1ccc2c(c1)ccc1ccccc12</smiles>

172<smiles>CCCCCCCCCCC(C(=O)c1ccc(OC)cc1)n1ccnc1</smiles>

173<smiles>CCC(C)C1CCCC1=O</smiles>

174<smiles>C1OC2OC12</smiles>

175<smiles>O=C1CCCCN1CCCCBr</smiles>

176<smiles>CCCCCCC(CO)CCCCCC</smiles>

$\mathrm{HO}$ 178<smiles>CCOC1CC(O)OC(C)C1O</smiles>

180

Fig. 12 continued 
<smiles>C=C1CC(C)(C)C2=CC(=O)OC2(C)C1</smiles>

181<smiles>CC(=O)/C=C/C1(O)C(C)=CC(=O)CC1(C)C</smiles>

182<smiles>O=C(O)CCC(=O)O</smiles>

183<smiles>C=C1C(=O)OC2C1CCC(C)C1(O)C(=O)C=CC21C</smiles>

184<smiles>CC1=CC(=O)CC(C)(C)C1(O)/C=C/C(C)O</smiles>

185<smiles>CC1=C(/C=C/CO)C(C)CC(O)C1O</smiles>

188<smiles>COc1cc(C2OCC3C(=O)OCC32)cc(OC)c1O</smiles>

186<smiles>CC1=CC(=O)CC(C)C1(O)/C=C/C(O)CO</smiles>

189<smiles>COc1cc(CC2COC(c3ccc(O)c(OC)c3)C2CO)ccc1O</smiles>

187

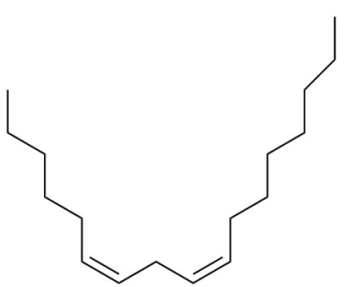

190<smiles>CCCCCC1(O)OC(=O)C(C)=C1C</smiles>

191<smiles>CCCCCCCCCCCCCCCC(=O)OCC(O)CO</smiles>

193<smiles></smiles>

194

Fig. 12 continued

expression of $\mathrm{Sp} 1$. In addition, treatment of the cells with MEDC (40 and $60 \mu \mathrm{g} / \mathrm{mL}$ ) decreased Mcl-1 expression, which is a downstream target of $\mathrm{Sp} 1$, indicating that $D$. chinensis contains natural bioactive products that induce the apoptosis of tumor cells overexpressing Sp1 [57].

$\mathrm{Li}$ et al. analyzed the petroleum ether extract of D. superbus by MTT colorimetry and isolated eight components, which were found to have antitumor activity at $100 \mathrm{mg} / \mathrm{L}$ against HeLa, Smmc-7721, HepG2, SK-hep1, A549, and Bel-7402 cell lines. The fractions with a strong antitumor activity were analyzed using GC-MS, and their main chemical components were fatty acid esterification derivatives and phenolic compounds. These findings provide a scientific basis for the 
Table 3 Compounds isolated from D. superbus and D. chinensis

\begin{tabular}{|c|c|c|c|c|}
\hline Classification & No & Compounds & Source & Refs \\
\hline \multirow[t]{25}{*}{ Saponins } & 1 & 3-O- $\beta$-D-Glucopyranosylolean-9(11),12-diene-23,28-dioicacid28-O- $\beta$-D-glucopyranoside & D. superbus & [11] \\
\hline & 2 & 3-O- $\beta$-D-Glucopyranosylolean-11,13(18)-diene-23,28-dioicacid28-O- $\beta$-D-glucopyranoside & D. superbus & {$[11,13]$} \\
\hline & 3 & $\begin{array}{l}\text { 3-O- } \beta \text {-D-Glucopyranosyl gypsogenic acid 28-O-[ } \beta \text {-D-6-O-((3S)-3-hydroxyl-3- methylglutaryl) } \\
\text { glucopyranosyl( } 1 \rightarrow 6)]-\beta \text {-D-glucopyranoside }\end{array}$ & D. superbus & [12] \\
\hline & 4 & $\begin{array}{l}\text { 3-O- } \beta \text {-D-Glucopyranosyl gypsogenic acid 28-O-[ } \beta \text {-D-Glucopyranosyl( }(\rightarrow 3)][\beta-\mathrm{D}-6-\mathrm{O}-((3 S)- \\
\text { hydroxyl-3-methylglutaryl)glucopyranosyl( }(1 \rightarrow 6)]-\beta \text {-D-glucopyranoside }\end{array}$ & D. superbus & {$[12]$} \\
\hline & 5 & 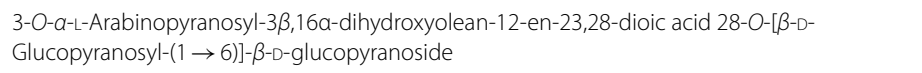 & D. superbus & {$[12]$} \\
\hline & 6 & Asiatic acid & D. superbus & [14] \\
\hline & 7 & Dianchinenoside A & D. chinensis & {$[15]$} \\
\hline & 8 & Dianchinenoside B & D. chinensis & {$[15]$} \\
\hline & 9 & Dianchinenoside C & D. chinensis & [17] \\
\hline & 10 & Dianchinenoside D & D. chinensis & [17] \\
\hline & 11 & Dianchinenoside E & D. chinensis & {$[16]$} \\
\hline & 12 & Dianchinenoside F & D. chinensis & {$[16]$} \\
\hline & 13 & Dianchinenoside G & D. chinensis & {$[16]$} \\
\hline & 14 & Dianchinenoside $\mathrm{H}$ & D. chinensis & {$[16]$} \\
\hline & 15 & 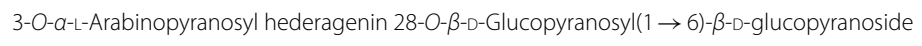 & D. chinensis & [24] \\
\hline & 16 & Madecassic acid & D. superbus & [19] \\
\hline & 17 & Sterculin A & D. superbus & {$[18]$} \\
\hline & 18 & (24R)-Cycloart-25-ene-3ß,24-diol & D. superbus & {$[18]$} \\
\hline & 19 & (24S)-Cycloart-25-ene-3ß,24-diol & D. superbus & [18] \\
\hline & 20 & $\beta$-Spinasterol & D. superbus & [18] \\
\hline & 21 & Stigmast-7-en-3 $\beta$-ol & D. superbus & [18] \\
\hline & 22 & $\beta$-Sitosterol glucoside & D. superbus & [38] \\
\hline & 23 & Hainanenside & D. superbus & [20] \\
\hline & 24 & 3-O- $\beta$-D-Glucopyranosyl olean-11, 13 (18)-diene-23, 28-dioic acid & D. superbus & [20] \\
\hline & 25 & Quillaic acid & D. superbus & [21] \\
\hline \multirow[t]{23}{*}{ Flavonoids } & 26 & Quercetin-3-O-glucoside & D. superbus & [22] \\
\hline & 27 & Quercetin-7-O-glucoside & D. superbus & [27] \\
\hline & 28 & Isoorientin & D. superbus & [23] \\
\hline & 29 & Orientin & D. superbus & [23] \\
\hline & 30 & 5-Hydroxy-7,5',4'-trimethoxyflavanone & D. superbus & [18] \\
\hline & 31 & 5,3'-Dihydroxy-7,4'-dimethoxyflavanone & D. superbus & [18] \\
\hline & 32 & 5,4'-Dihydroxy-7,3'-dimethoxyflavanone & D. superbus & [18] \\
\hline & 33 & Isoorientin-2"-O-glucoside & D. chinensis & [24] \\
\hline & 34 & Chrysoeriol-7-O-glucoside & D. chinensis & [24] \\
\hline & 35 & Isovitexin-2"-O-glucoside & D. chinensis & [24] \\
\hline & 36 & 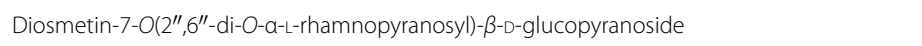 & D. superbus & {$[28,42]$} \\
\hline & 37 & Quercetin-3-O-rutinoside & D. superbus & {$[30]$} \\
\hline & 38 & Isorhamnetin-3-O-glucoside & D. superbus & [30] \\
\hline & 39 & Quercetin-3-O-rhamnoside 7-O-rhamnoside & D. superbus & [30] \\
\hline & 40 & Kaempferol-3-O-glucoside-glucoside 7-O-rhamnoside & D. superbus & [30] \\
\hline & 41 & Quercetin-3-O-rhamnoside-glucoside 7-O-rhamnoside & D. superbus & [30] \\
\hline & 42 & Luteolin & D. superbus D. chinensis & {$[23,25,29]$} \\
\hline & 43 & 3,5,7-Trihydroxy-3',5'-dimethoxylflavone & D. superbus & {$[26]$} \\
\hline & 44 & Kaempferol & D. superbus & {$[26]$} \\
\hline & 45 & Quercetrin & D. superbus & [26] \\
\hline & 46 & Tricin & D. superbus & [31] \\
\hline & 47 & Diosmetin & D. superbus & [31] \\
\hline & 48 & 5-Hydroxy-7,3'4'-trimethoxydihydroflavone & D. superbus & [20] \\
\hline \multirow[t]{2}{*}{ Peptides } & 49 & Dianthin A & D. superbus & [33] \\
\hline & 50 & Dianthin B & D. superbus & [33] \\
\hline
\end{tabular}


Table 3 (continued)

\begin{tabular}{|c|c|c|c|c|}
\hline Classification & No & Compounds & Source & Refs \\
\hline & 51 & Dianthin C & D. superbus & [34] \\
\hline & 52 & Dianthin D & D. superbus & [34] \\
\hline & 53 & Dianthin E & D. superbus & [34] \\
\hline & 54 & Dianthin F & D. superbus & [34] \\
\hline & 55 & Dianthin G & D. superbus & [35] \\
\hline & 56 & Dianthin $\mathrm{H}$ & D. superbus & [35] \\
\hline & 57 & Dianthin I & D. chinensis & {$[37,45]$} \\
\hline & 58 & Pseudostellarin A & D. chinensis & {$[37,45]$} \\
\hline & 59 & Heterophyllin J & D. chinensis & {$[37,45]$} \\
\hline & 60 & 4-Methoxydianthramide B & D. superbus & [34] \\
\hline & 61 & Cyclo-(L-N-methyl Glu-L-N-methyl Glu & D. chinensis & [36] \\
\hline & 62 & Cyclo-(L-methyl Glu-ester-L-methyl Glu ester & D. chinensis & [36] \\
\hline & 63 & Cyclo-(L-methyl Glu-ester-L-Glu) & D. chinensis & [36] \\
\hline & 64 & Cyclo-(L-Glu-L-Glu) & D. chinensis & [36] \\
\hline & 65 & Tyrocidine B & D. superbus & [30] \\
\hline \multirow[t]{6}{*}{ Anthraquinones } & 66 & Emodin & D. superbus & {$[19,25,38,40]$} \\
\hline & 67 & Physcion & D. superbus & {$[38,39]$} \\
\hline & 68 & Emodin-8-O- $\beta$-D-glucopyranoside & D. superbus & {$[25,38]$} \\
\hline & 69 & Physcion-8-O- $\beta$-D-glucoside & D. superbus & [20] \\
\hline & 70 & MelrubiellinA & D. superbus & [21] \\
\hline & 71 & MelrubiellinB & D. superbus & [21] \\
\hline \multirow[t]{15}{*}{ Phenolic acids } & 72 & p-Hydroxybenzoic acid & D. superbus & {$[19,41]$} \\
\hline & 73 & 3-Hydroxy-4-methoxybenzoic acid & D. superbus & [19] \\
\hline & 74 & Methyl 3,4-dihydroxybenzoate & D. superbus & [38] \\
\hline & 75 & Methyl 3-(3,4-dihydroxyphenyl) propionate & D. superbus & [38] \\
\hline & 76 & 4-hydroxy-3-methoxy-pentyl ester benzenepropanoic acid & D. superbus & [42] \\
\hline & 77 & Vanillic acid & D. superbus & [42] \\
\hline & 78 & 4-Hydroxy-benzeneacetic acid & D. superbus & [42] \\
\hline & 79 & Hydroferulic acid & D. superbus & [42] \\
\hline & 80 & Methyl hydroferulate & D. superbus & [42] \\
\hline & 81 & (E)-methyl-4-hydroxy-4-(8a-methyl-3-oxodecahydronaphthalen-4a-yl) & D. superbus & [42] \\
\hline & 82 & Trans-p-coumaric acid & D. superbus & [26] \\
\hline & 83 & Catechol & D. superbus & [21] \\
\hline & 84 & Salicylic acid & D. superbus & [21] \\
\hline & 85 & $\beta$-hydroxypropiovanillone & D. superbus & [31] \\
\hline & 86 & 4-Methoxyphenylacetic acid & D. superbus & [20] \\
\hline \multirow[t]{6}{*}{ Amides } & 87 & Patriscabratine & D. superbus & [19] \\
\hline & 88 & $N$-p-coumarylanthranilic acid & D. superbus & [19] \\
\hline & 89 & Methoxydianthramide S & D. superbus & [26] \\
\hline & 90 & 2-[(2,4-Dihydroxybenzoyl) amino]-4-methoxy-benzoic acid & D. superbus & [26] \\
\hline & 91 & Aurantiamide & D. superbus & [21] \\
\hline & 92 & Aurantiamide acetate & D. superbus & [31] \\
\hline \multirow[t]{9}{*}{ Phenylpropanoids } & 93 & 4-Methoxy-benzeneacetic acid & D. superbus & [42] \\
\hline & 94 & Scopoletin & D. superbus & [26] \\
\hline & 95 & 6-Methoxy-hydroxycoumarin & D. superbus & [26] \\
\hline & 96 & Epipinoresinol & D. superbus & [21] \\
\hline & 97 & Pinoresinol & D. superbus & [21] \\
\hline & 98 & Prinsepiol & D. superbus & [21] \\
\hline & 99 & Medioresinol & D. superbus & [31] \\
\hline & 100 & Syringaresinol & D. superbus & [31] \\
\hline & 101 & Dehydrodiconiferyl alcohol & D. superbus & [31] \\
\hline \multirow[t]{2}{*}{ Others } & 102 & Hydoxydihydrobovolide & D. superbus & [18] \\
\hline & 103 & Cis-3-hexen-1-ol & D. superbus & [43] \\
\hline
\end{tabular}


Table 3 (continued)

\begin{tabular}{|c|c|c|c|c|}
\hline Classification & No & Compounds & Source & Refs \\
\hline & 104 & Phenylethane & D. superbus & [43] \\
\hline & 105 & $N$-hexanol & D. superbus & {$[43]$} \\
\hline & 106 & Sorbic Acid & D. superbus & {$[43]$} \\
\hline & 107 & Benzaldehyde & D. superbus & [43] \\
\hline & 108 & N-heptanol & D. superbus & [43] \\
\hline & 109 & 1-Octen-3-ol & D. superbus & [43] \\
\hline & 110 & 6-Methyl-5-hepten-2-one & D. superbus & [43] \\
\hline & 111 & 2-Methyl-2-hepten-6-ol & D. superbus & {$[43]$} \\
\hline & 112 & Phenylacetaldehyde & D. superbus & {$[43]$} \\
\hline & 113 & $p$-tolualdehyde & D. superbus & [43] \\
\hline & 114 & N-octanol & D. superbus & [43] \\
\hline & 115 & $(E, E)$-3,5-octadien-2-one & D. superbus & [43] \\
\hline & 116 & Linalool & D. superbus & [43] \\
\hline & 117 & 1-Acetyl-2-methylcyclopentene & D. superbus & [43] \\
\hline & 118 & $\mathrm{~N}$-nonanal & D. superbus & {$[43]$} \\
\hline & 119 & $N$-nonanol & D. superbus & {$[43]$} \\
\hline & 120 & decanal & D. superbus & {$[43]$} \\
\hline & 121 & n-decanol & D. superbus & [43] \\
\hline & 122 & $a$-terpineol & D. superbus & [43] \\
\hline & 123 & $a$-cyclocitral & D. superbus & [43] \\
\hline & 124 & Cis-chrysanthenylacetate & D. superbus & [43] \\
\hline & 125 & $(E)$-anethole & D. superbus & {$[43]$} \\
\hline & 126 & a-Damascenone & D. superbus & {$[43]$} \\
\hline & 127 & Tetrahydrogeranyl acetone & D. superbus & [43] \\
\hline & 128 & Geranyl acetone & D. superbus & [43] \\
\hline & 129 & 2,6,10-trimethyltetradecane & D. superbus & [43] \\
\hline & 130 & $\beta$-lonone & D. superbus & [43] \\
\hline & 131 & 3,7,11-Trimethyl-1-dodecanol & D. superbus & {$[43]$} \\
\hline & 132 & 2-Methylhexadecan-1-ol & D. superbus & {$[43]$} \\
\hline & 133 & 6,10,14-Trimethyl-2-pentadecanone & D. superbus & [43] \\
\hline & 134 & Farnesy lacetone & D. superbus & {$[43]$} \\
\hline & 135 & Palmitic acid & D. superbus & [43] \\
\hline & 136 & Cis-phytol & D. superbus & [43] \\
\hline & 137 & (E)-4-Methoxycinnamic acid & D. superbus & {$[42]$} \\
\hline & 138 & 3-Methoxy-4-hydroxyphenylethanol & D. superbus & {$[42]$} \\
\hline & 139 & Loliolide & D. superbus & {$[26]$} \\
\hline & 140 & 1-Monopalmitin & D. superbus & {$[26]$} \\
\hline & 141 & 2,4-Dimethylhexane & D. superbus & [44] \\
\hline & 142 & 2,4-Di-tert-butylphenol & D. superbus & {$[44]$} \\
\hline & 143 & Methyl 12-methyltridecanoate & D. superbus & [44] \\
\hline & 144 & Tetradecane & D. superbus & {$[44]$} \\
\hline & 145 & Methyl 13-methylpentadecanoate & D. superbus & [44] \\
\hline & 146 & 3,7,11,15-Tetramethyl-1-hexadecadiene-3-ol & D. superbus & {$[44]$} \\
\hline & 147 & 16-methylheptadecanoic acid methyl ester & D. superbus & [44] \\
\hline & 148 & 2,4-dimethylbenzaldehyde & D. superbus & {$[44]$} \\
\hline & 149 & 2,4,6-trimethyloctane & D. superbus & {$[44]$} \\
\hline & 150 & 2-Methyldecane & D. superbus & {$[44]$} \\
\hline & 151 & 2,6,11-trimethyldodecane & D. superbus & {$[44]$} \\
\hline & 152 & 2,6-di-tert-butylphenol & D. superbus & [44] \\
\hline & 153 & Methyl-3-(3,5-di-tert-butyl-4-hydroxyphenyl) propionate & D. superbus & [44] \\
\hline & 154 & (Z)-9-Octadecenylamide & D. superbus & {$[44]$} \\
\hline & 155 & 2,4-Nondiene & D. superbus & [44] \\
\hline & 156 & 7,9-Di-tert-butyl-1-oxaspiro $(4,5)$ deca-6,9-diene-2,8-dione & D. superbus & {$[44]$} \\
\hline
\end{tabular}


Table 3 (continued)

\begin{tabular}{|c|c|c|c|c|}
\hline Classification & No & Compounds & Source & Refs \\
\hline & 157 & Methyl palmitate & D. superbus & [44] \\
\hline & 158 & 11,13-dimethyl-12-tetradecene-1-ol acetate & D. superbus & [44] \\
\hline & 159 & (R)-4-[(R, E)-3-hydroxy-1-butenyl]-3,5,5-trimethyl-2-cyclohexene-1-one & D. superbus & [44] \\
\hline & 160 & 1-Hydroxy-4-keto-2-ionone & D. superbus & [44] \\
\hline & 161 & Dibutyl phthalate & D. superbus & [44] \\
\hline & 162 & 4-Methyl-1-undecanone & D. superbus & [44] \\
\hline & 163 & 3, 3-Dimethylhexane & D. superbus & [44] \\
\hline & 164 & 2-Ethyl-1-heptanol & D. superbus & [44] \\
\hline & 165 & 1-Pentanol & D. superbus & [44] \\
\hline & 166 & 2-n-Pentylfuran & D. superbus & [44] \\
\hline & 167 & 4-Methyldocosane & D. superbus & [44] \\
\hline & 168 & 1-Hexadecene & D. superbus & [44] \\
\hline & 169 & Cis-7-decene aldehyde & D. superbus & [44] \\
\hline & 170 & 2,6-Di-tert-butyl-p-benzoquinone & D. superbus & [44] \\
\hline & 171 & Dimethyl azelaite & D. superbus & [44] \\
\hline & 172 & Phenanthrene & D. superbus & [44] \\
\hline & 173 & 2-(1H-imidazol-1-yl)-1-(4-methoxyphenyl)-1-dodecanone & D. superbus & [44] \\
\hline & 174 & 2-Sec-butyl Cyclopentanone & D. superbus & [44] \\
\hline & 175 & 3,4-Epoxytetrahydrofuran & D. superbus & [44] \\
\hline & 176 & 2-Oxo-1-(4-bromo- $n$-butyl) piperidine & D. superbus & [44] \\
\hline & 177 & 2-Hexyl-1-octanol & D. superbus & [44] \\
\hline & 178 & Methyl hexadecanoate & D. superbus & [44] \\
\hline & 179 & 1-Octacosanol & D. superbus & [44] \\
\hline & 180 & L-Dianose & D. chinensis & [44] \\
\hline & 181 & Dehydrololiolide & D. superbus & [21] \\
\hline & 182 & Dehydrovomifoliol & D. superbus & [21] \\
\hline & 183 & Succinic acid & D. superbus & [21] \\
\hline & 184 & Parthenin & D. superbus & [31] \\
\hline & 185 & Vomifoliol & D. superbus & [31] \\
\hline & 186 & Zhebeiresinol & D. superbus & [31] \\
\hline & 187 & Lariciresinol & D. superbus & [31] \\
\hline & 188 & |xerol B & D. superbus & [31] \\
\hline & 189 & Cucumegastigmanes I & D. superbus & [31] \\
\hline & 190 & $(6 z, 9 z)$-heptadecadiene & D. superbus & [31] \\
\hline & 191 & 3,6, 9-nonadecatriene & D. superbus & [21] \\
\hline & 192 & 5-Hydroxyl-3,4-dimethy-5-pentyl-2(5H)-furanone & D. superbus & [20] \\
\hline & 193 & 1-Glycerol palmitate & D. superbus & [20] \\
\hline & 194 & 3-(3,','-Dihydroxyphenyl) lactic acid sodium salt & D. superbus & [20] \\
\hline
\end{tabular}

clinical use of Dianthi herba in the treatment of tumor diseases [44].

In conclusion, extracts of the effective parts of Dianthi herba have antitumor biological activity, which is of importance in explaining the material basis of the antitumor efficacy of Dianthi and provides evidence for the clinical use of Dianthus spp. in the treatment of tumor. However, it is necessary to strengthen research on the active ingredients and anticancer activity of Dianthi in vivo to provide a theoretical basis for the development and application of Dianthi.

\section{Antioxidant activity}

Yun et al. studied the effect of Dunhuang Yifang Qumai decoction (20,10, and $5 \mathrm{~g} / \mathrm{kg}$, respectively) on the nuclear factor erythroid-2 related factor 2 (Nrf2)/antioxidant response element (ARE) signaling pathway in a rat renal calcium oxalate stone model, dosed by gavage with $1 \%$ ethylene glycol and 2\% ammonium chloride solution [58]. Superoxide dismutase activity, malondialdehyde level, and total antioxidant capacity in rat serum were evaluated using hematoxylin and eosin staining. The pathomorphological changes in the kidney tissue of rats in 


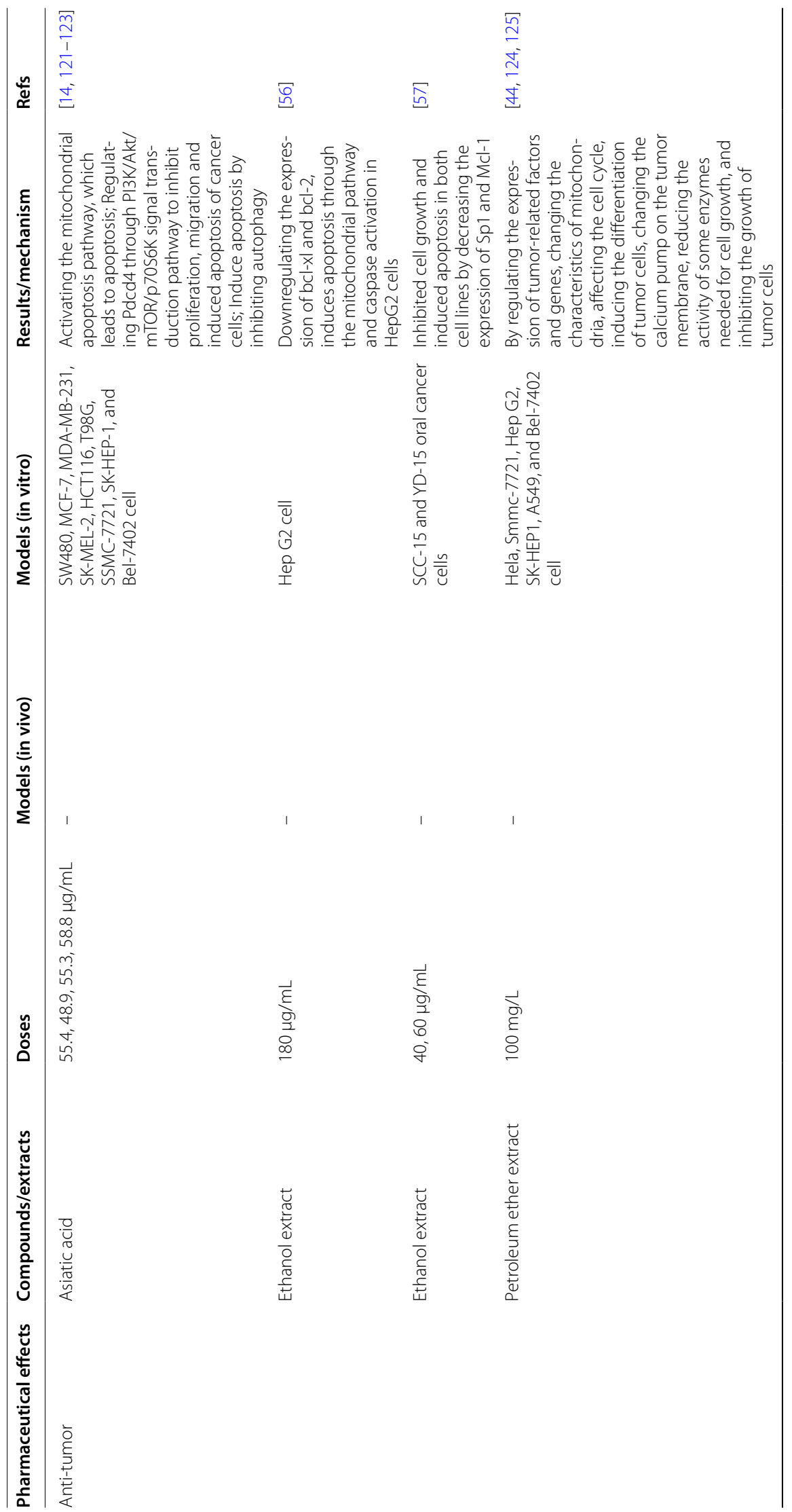




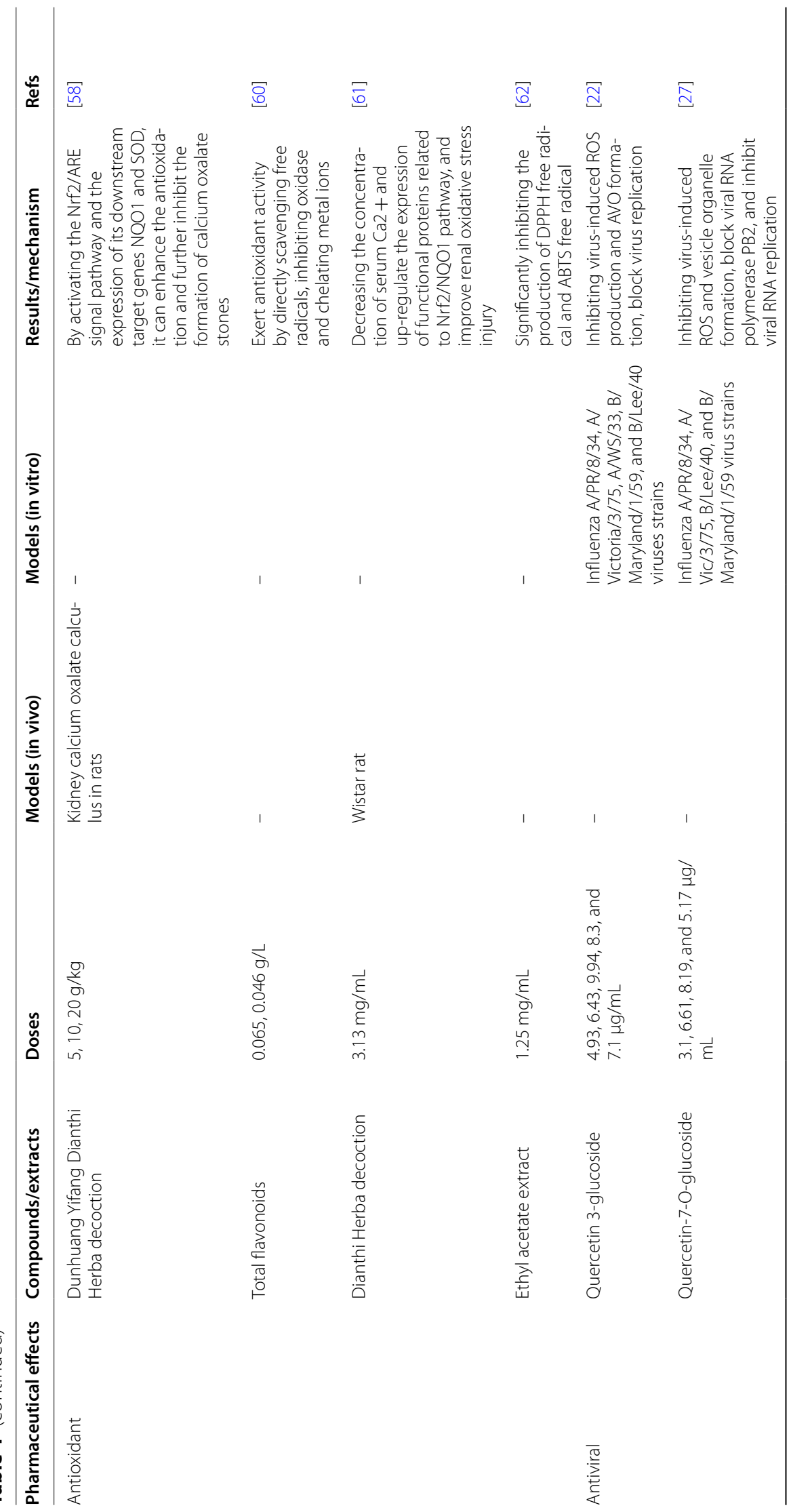




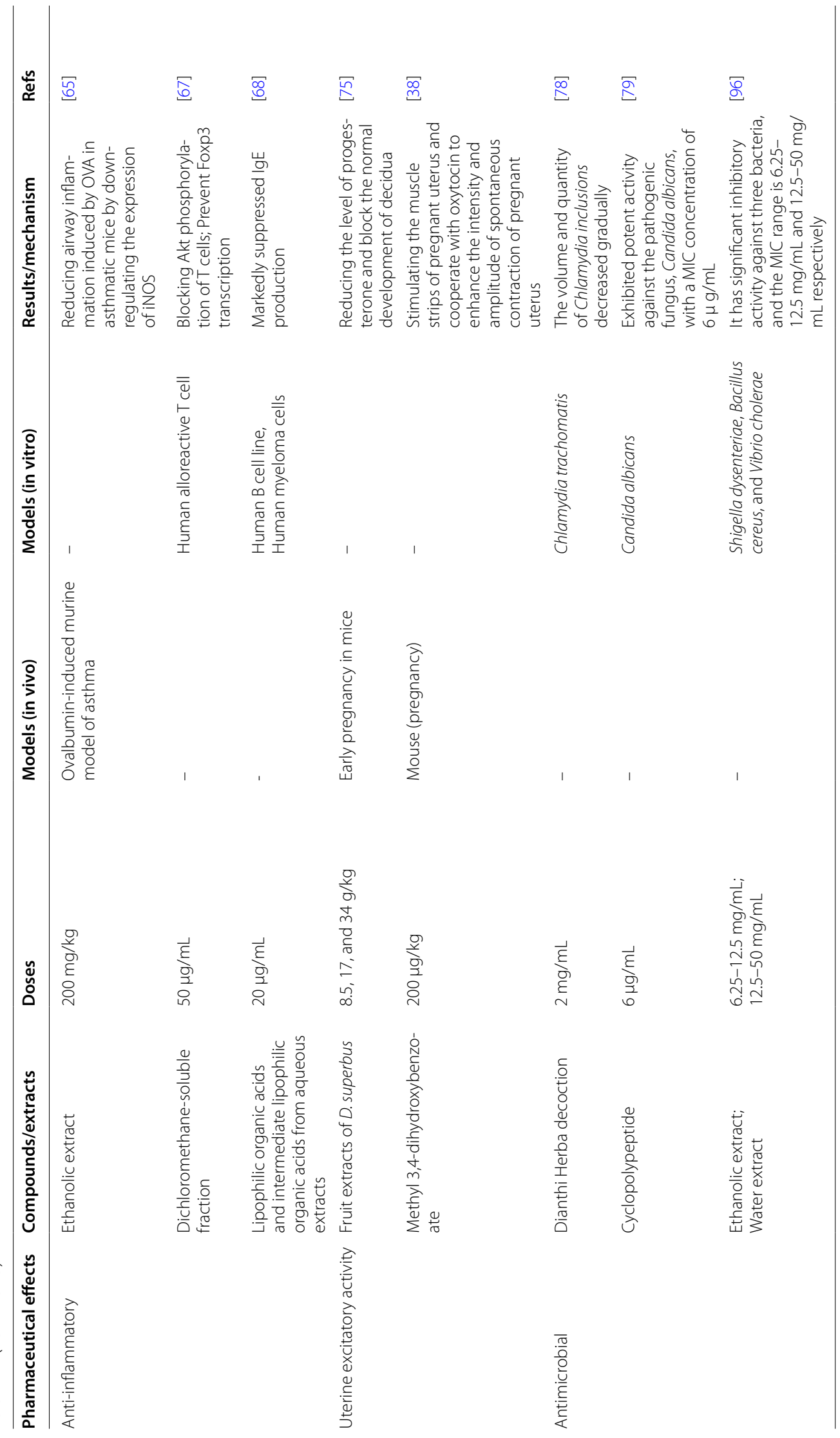


each group were examined. In addition, the kidney tissue expression of Nrf2 and NAD(P)H:quinone oxidoreductase 1 (NQO1) mRNA was determined using RT-PCR; the protein expression of Nrf2 and ARE evaluated using immunohistochemistry. The results showed oxidative stress injury in the treatment groups that were administered high-, medium-, and low-doses of the Dunhuang medical prescription Qumai decoction, and its improving effect was dose-dependent [58]. The mechanism of the therapeutic action of Qumai decoction may involve the inhibition of kidney stone formation by upregulating the expression of factors related to the Nrf2/ARE signaling pathway, which provides a basis for its clinical use of Qumai in the prevention and treatment of kidney stones.

Flavonoids extracted from $D$. superbus using solid fermentation of Aspergillus niger were evaluated for antioxidant properties in vitro using the hydroxyl radical scavenging assay. The results showed that the scavenging ability increased in a concentration-dependent manner [59]. The $\mathrm{IC}_{50}$ value of the flavonoid against the hydroxyl radical was statistically determined to be $0.065 \mathrm{~g} / \mathrm{L}$. At concentrations of $0.05-0.20 \mathrm{~g} / \mathrm{L}$, the free radical scavenging ability of the buckwheat flavonoid increased in a concentration-dependent manner. The $\mathrm{IC}_{50}$ value for free radical scavenging was $0.046 \mathrm{~g} / \mathrm{L}$, indicating that $D$. superbus has a strong antioxidant activity [60].

In addition, Chen et al. used a Wistar rat model to examine the effect of Qumai decoction on lipid peroxidation in liver homogenates [82]. At a concentration of $3.13 \mathrm{mg} / \mathrm{mL}$, the decoction had obvious inhibitory effects on lipid peroxidation in rat liver homogenates $(P<0.001)$ [61]. The antioxidant activity of components of different solvent fractions of the extract of D. superbus was studied, and each component exhibited varying inhibitory effects on free radicals. Components of the ethyl acetate (EtOAc) fractions exhibited a more potent effect than those of other fractions, and the $\mathrm{IC}_{50}$ indicated a strong antioxidant activity at $1.25 \mathrm{mg} / \mathrm{mL}$ [97, 98]. Furthermore, the scavenging effect of all components of the EtOAc fraction on hydroxyl radicals was significant [62].

The antioxidant activity of Dianthus is exerted through different mechanisms by scavenging free radicals and by upregulating factors related to the Nrf2/ARE signaling pathway; these findings provide a preliminary basis for the development of new natural antioxidants and the further development of anti-aging drugs [63, 64]. Therefore, although the antioxidant activity of $D$. superbus has been widely studied, it is necessary to further study the mechanism through which it reduces reactive oxygen species (ROS) and free radical formation, to provide a detailed theoretical basis for the practical application of D. superbus.

\section{Antiviral activity}

Nile et al. studied the antiviral and cytotoxic activities of quercetin 3-glucoside (Q3G) isolated from D. superbus against influenza virus infection [22]. To determine the mechanism underlying the antiviral effect of Q3G on the influenza virus, time-dependent antiviral tests, molecular docking studies, virus-induced symptom analysis, pre-incubation, and screening of related gene expression were conducted [99]. Q3G from D. superbus showed strong antiviral activity against influenza $A$ and $B$ viruses. The $\mathrm{IC}_{50}$ value against $\mathrm{A} / \mathrm{PR} / 8 / 34, \mathrm{~A} /$ Victoria/ $3 / 75, \mathrm{~A} /$ WS/33, B/Maryland/1/59, and B/Lee/40 was $4.93,6.43$, 9.94, 8.3, and $7.1 \mu \mathrm{g} / \mathrm{mL}$, respectively. It inhibited virusinduced cellular ROS generation and acidic vesicular organelles formation. Moreover, Q3G and oseltamivir, administered to the control group, were found to be cytotoxic [22]. The half-maximal cytotoxic concentration $\left(\mathrm{CC}_{50}\right)$ value was $>100 \mu \mathrm{g} / \mathrm{mL}$ and nontoxic. In addition, Q3G did not inhibit the neuraminidase (NA) activity of the influenza virus but blocked viral replication, showing a more competitive binding affinity $(-8.0 \mathrm{kcal} / \mathrm{mol})$ than guanosine triphosphate (GTP) $(-7.0 \mathrm{kcal} / \mathrm{mol})$ in blocking the $\mathrm{Pb} 2$ subunit of influenza viral polymerase. Therefore, Q3G has a positive protective effect on infected host cells, and a strong inhibitory effect on influenza A and B viruses, which provides a new research direction on Q3G in the development of anti-influenza drugs.

Quercetin-7-O-glucoside (Q7G), a compound isolated from $D$. superbus, was analyzed for inhibitory effects on viral RNA replication using quantitative RT-PCR. The blocking effect of Q7G on the basic protein subunits of the RNA polymerase of influenza virus was detected using the AutoDock Vina program and M7GTP using a computer molecular docking assay. Q7G showed no cytotoxicity in MDCK cells $\left(\mathrm{CC}_{50}>100 \mu \mathrm{g} / \mathrm{mL}\right)$, and its $\mathrm{IC}_{50}$ value was $3.1,6.61,8.19$, and $5.17 \mu \mathrm{g} / \mathrm{mL}$ against influenza A/PR/8/34, A/Vic/3/75, B/Lee/40, and B/Maryland/1/59 virus strains, respectively. Treatment with $\mathrm{Q} 7 \mathrm{G}$ highly reduced ROS and autophagy formation induced by influenza virus infection. Q7G does not bind directly to virus particles or affect NA activity. These results indicate that Q7G inhibits RNA polymerase and occupies the binding site of M7GTP on Pb2 [27]. In conclusion, $D$. superbus has strong antiviral activity as a TCM, especially the active compounds Q3G and Q7G. These findings provide a direction for further research on $D$. superbus as an antiviral drug.

\section{Anti-inflammatory activity}

Shin et al. evaluated the anti-inflammatory effects of $D$. superbus fructus ethanolic extract (DSE) $(200 \mathrm{mg} / \mathrm{kg})$ 
on Th2-type cytokines, eosinophil infiltration, and other factors in an ovalbumin (OVA)-induced, murine asthma model [65]. To elucidate the potential mechanism of the anti-inflammatory effect of DSE, the expression of inducible nitric oxide synthase (iNOS) in the respiratory tract was also analyzed. DSE significantly reduced the levels of interleukin (IL)-4, IL-13, eotaxin, and immunoglobulin $\mathrm{E}$ (IgE); the number of inflammatory cells in the bronchoalveolar lavage fluid (BALF); level of inflammatory cell infiltration; and mucus production in the respiratory tract [100]. The results indicate that DSE also attenuates the overexpression of iNOS induced by OVA challenge. This suggests that DSE effectively protects against allergic airway inflammation by downregulating iNOS expression, and it has potential as a therapeutic agent for allergic asthma $[65,66]$.

In another study, to discover novel immunosuppressants, cytokine enzyme-linked immunosorbent spot and enzyme-linked immunosorbent assays were used to verify the dichloromethane soluble component of $D$. superbus $(50 \mu \mathrm{g} / \mathrm{mL})$ for suppressive effects against human alloreactive $\mathrm{T}$ cells [101]. The addition of $D$. superbus extracts to human mixed lymphocyte cultures caused a dose-dependent inhibition of proliferation and interferon $c$ (IFNc) production by memory alloreactive $\mathrm{T}$ cells and increased the proportion of forkhead box P3-positive $\left(\right.$ Foxp $^{+}{ }^{+} \mathrm{CD}^{+} \mathrm{T}$ cells. To determine whether the extracts of $D$. superbus induce regulatory $\mathrm{T}$ cells, anti CD3/CD28-stimulated CD4 T cells were treated, and a dose-dependent upregulation of Foxp3 was found to be associated with novel suppressive effects. Mechanistically, extracts of $D$. superbus did not induce T cell IL-10 or transforming growth factor beta (TGF $\beta$ ), but they blocked T cell protein kinase B (Akt) phosphorylation, a key signaling nexus required for $\mathrm{T}$ cell proliferation and expansion that simultaneously prevents Foxp 3 transcription. This study provides novel insights into the antiinflammatory effects of D. superbus [67].

Slotkin et al. reported that lipophilic organic acids $(20 \mu \mathrm{g} / \mathrm{mL})$ and intermediate lipophilic organic acids $(20 \mu \mathrm{g} / \mathrm{mL})$ in aqueous extracts of D. superbus were highly effective in reducing IgE secretion by a human $B$ cell line. Furthermore, preliminary in vivo studies showed that $D$. superbus reduced the symptoms of anaphylactic shock in peanut-sensitized mice [68].

Although basic animal studies are still needed to investigate the anti-inflammatory effect of D. superbus, its anti-inflammatory effect and potential action mechanism provide a basis for its potential application in the development of new drugs. In addition, its anti-inflammatory activity can be enhanced when it is used in combination with other drugs as a combined supplement [69].

\section{Diuretic activity}

In selected hospitals, from 2007 to 2014, Xu et al. treated 200 patients with ureteral calculi who were allocated to the control and treatment groups using the random number table method [70], with 100 patients in each group. The patients in the control group were administered extracorporeal shock wave lithotripsy combined with rehydration, spasmolysis, and diuretic therapy. The results suggested that $D$. superbus decoction combined with extracorporeal shock wave lithotripsy for the treatment of ureteral calculi required a short treatment time and that the patients exhibited a low recurrence rate. This finding suggests that this treatment may be suitable for widespread clinical application [70]. Li et al. gavaged rabbits with Qumai decoction ( $2 \mathrm{~g} / \mathrm{kg}$ ), and after $60 \mathrm{~min}$, the urinary output increased significantly, demonstrating an improved diuretic effect [71]. Deng et al. prepared a compound decoction containing $D$. superbus, and the effect was investigated using laboratory examinations such as routine urine analysis as well as clinical symptom evaluation. The results showed that the urinary tract symptoms were relieved following treatment with the decoction [72]. Other studies have proven that prescription drugs containing $D$. superbus exhibit diuretic effects that improve urinary tract symptoms [71, 73, 74].

\section{Uterine excitatory activity}

$\mathrm{Li}$ et al. used a mouse model of early gestation to evaluate the effects of various doses of fruit extracts of $D$. superbus on embryo number and growth [75]. Mice in the gestation period were intragastrically administered various doses $(8.5,17$, and $34 \mathrm{~g} / \mathrm{kg})$ of the extract once a day, and fruit extracts of $D$. superbus showed obvious effects on early pregnancy in mice. The fruit extract of $D$. superbus at the three doses increased the abortion rate in a dose-dependent manner. The anti-pregnancy effects of the fruit may be mediated by a reduction in pregnancy ketone levels, which prevents normal development of the decidua [75, 76]. Wang et al. acquired methyl 3,4-dihydroxybenzoate from $D$. superbus, using an in vivo mouse (pregnancy) uterus spontaneous life record as a model [38]. The result indicated that $200 \mu \mathrm{g} / \mathrm{kg}$ methyl 3,4-dihydroxybenzoate excited the pregnant uterus, which was monitored by uterine smooth muscle contraction strength and amplitude [38]. Guo et al. used in vitro rat and in vivo rabbit uterus models to investigate the synergistic effects of the extracts and prostaglandin E2 [77]. The experimental results indicated that alcohol extracts excited uterine smooth muscles more when combined with the drug, and the degree of contraction in the treatment group $(4.33 \pm 2.19 \mathrm{~cm})$ was significantly higher than that before administration. Therefore, the ethanol extract 
of $D$. superbus has an obvious excitatory effect on the uterus of anesthetized free in vivo rats and the isolated peroneal muscle strips of rats. These studies show that Dianthi herba can stimulate the uterus, which is consistent with its traditional use in China to treat amenorrhea.

\section{Antimicrobial activity}

The antimicrobial activity of various Chinese herbs against Chlamydia trachomatis in the urogenital tract was tested in a previous study, and the results showed that Qumai decoction exhibits high sensitivity and a minimum inhibitory concentration (MIC) of $<2 \mathrm{mg} / \mathrm{mL}$. Furthermore, the volume and quantity of Chlamydia inclusions decreased gradually with increasing Qumai decoction concentration, and finally disappeared. Chlamydia trachomatis growth was found in the blank control pores, but not in the negative control wells [78]. Antimicrobial experiments with several strains were used to compare the plant-based cyclopolypeptide (XIII) from $D$. superbus with the standard drug, griseofulvin. Cyclopolypeptide (XIII) exhibited potent activity against the pathogenic fungus, Candida albicans, with an MIC of $6 \mu \mathrm{g} / \mathrm{mL}$ [79]. Moreover, the inhibitory effects of ethanol and water extracts of D. superbus on the activities of Shigella dysenteriae, Bacillus cereus, and Vibrio cholerae were studied. The MIC was $6.25-12.550 \mathrm{mg} / \mathrm{mL}$, and the minimum bactericidal concentration (MBC) was 12.525 $12.550 \mathrm{mg} / \mathrm{mL}$. The results showed that the ethanol and water extracts of $D$. superbus had positive inhibitory effects on S. dysenteriae, B. cereus, and V. cholerae [10].

These studies show that Dianthi herba has antibacterial effects, but most of these studies have been carried out in vitro, and it is necessary to use modern technology to study the potential mechanism of action and to translate the in vitro and in vivo research findings into clinical applications.

\section{Other effects}

Yun et al. isolated two bioactive compounds from $D$. superbus, 4-hydroxy-benzeneacetic acid and 4-methoxybenzeneacetic acid, to determine the components mediating its neuroprotective activity against glutamateinduced death of hippocampal neuronal HT22 cells. The two compounds effectively protected HT22 cells against glutamate toxicity [42]. Glutamate is obviously toxic to hippocampal neurons and was used to induce damage in the model used to determine the effects of $D$. superbus on cell viability. The cell viability was evaluated using the MTT assay. The EtOAc soluble fraction of D. superbus extract was obviously active, and it increased cell viability to $73.89 \%$ and $94.35 \%$ at concentrations of 10 and $100 \mu \mathrm{g} /$ $\mathrm{mL}$, respectively. These phenomena suggest that Dianthi herba may have neuroprotective activity.
Dianthi herba possesses several other biological activities such as hemolytic, anthelmintic, and antiallergic effects. In a previous study, a high concentration (100\%) of D. superbus alcoholic extract showed slight hemolytic effects and insect-repellent activity at a dose of $2 \mathrm{mg} / \mathrm{mL}$ [80]. Yoon et al. studied the protective effect of EtOAc extract of $D$. superbus in renal inflammation and fibrosis; the extract at a dose of 10 or $50 \mathrm{mg} / \mathrm{kg} / \mathrm{day}$ administered to $d b / d b$ mice for 8 weeks significantly improved the blood glucose and insulin levels, insulin resistance, homeostasis model assessment index, and HbA1c level. Therefore, the extract can be used as a potential drug to treat glomerulonephritis and glomerulosclerosis, which lead to diabetic nephropathy [81]. Zhang et al. found that Gualou Qumai decoction reduced the level of C-reactive protein (CRP), IL- 6 , and TGF $\beta 1$ in the renal tissue of rats and exerted the therapeutic effect of alleviating inflammatory reaction and renal fibrosis. In a previous study, Dianthi herba combined with Magnolia officinalis cortex, Aurantii fructus immaturus, and other medicines was used to treat pancreatic hydatoncus diseases; the results showed considerable clinical curative effects of the combination [1]. The extract of D. chinensis $(500 \mu \mathrm{g} /$ $\mathrm{mL}$ ) inhibited the production of IgE in vitro and in vivo in peanut-allergic mice, considerably reduced the allergic reaction caused by peanuts, and exerted a certain antiallergic effect [83].

In general, Dianthi herba has various pharmacological activities; however, screening and evaluation of its active compounds remain at the crude extract level. Research on its chemical composition, biological activity, pharmacodynamics, and mechanism of action remain limited. These gaps should be addressed in the future.

\section{Toxicity}

Currently, there are only a few reports on the toxicity of Dianthi herba. The suggested daily dose of Dianthi herba is 9-15 g, and pregnant women should use it cautiously, as documented in the Pharmacopoeia of the PRC (Pharmacopoeia Commission of PRC, 2020). A study on the antifertility and genotoxic effects of Dianthi herba decoction in pregnant mice has shown that doses of 10,15 , and $30 \mathrm{~g} / \mathrm{kg}$ considerably affected the implantation period and early pregnancy. Furthermore, 15 and $30 \mathrm{~g} / \mathrm{kg}$ Dianthi herba exerted certain mid-term pregnancy-terminating effect, which was apparent in mice in the late pregnancy stage, where it shortened the gestation period and reduced the weight of offspring. This is consistent with the "abortion and inferior occlusive blood" effects of Dianthi herba recorded in Shennong's Classic Materia Medica. Dianthi herba caused abortion in mice in the early pregnancy stage, indicating the lethal effect of this drug [84, 102]. In addition, 
Dianthi herba can cause damage to the kidney. Previously, different doses $(0.25,0.5$, and $2.5 \mathrm{~g} / \mathrm{mL})$ were used to determine the serum biochemistry of liver and kidney function. Organ index analysis and histopathology were performed to detect the morphological changes of the main organs in mice. qPCR was used to determine the role of organic anion transporters OAT1 and OAT3 in the toxic injury in mice treated with Dianthi herba. The results showed that the middle and high dose groups of Dianthi herba had different degrees of damage of the main organs in mice, especially the kidney was severely damaged $[103,104]$.

\section{Clinical applications}

Clinical and human studies have shown that Dianthi herba is mainly used in the treatment of urinary diseases, diabetic nephropathy, edema, cysts, esophageal tumors, and rectal cancer. In piglets and lamb with dysentery, Dianthi herba has a unique curative effect. In a recent study on chronic prostatitis, 60 patients with damp-heat, stasis type, chronic prostatitis were selected and randomly divided into two groups, with 30 patients in each group. The mean age of the control group was 31.1 years and the mean course of the disease was 33 months. A Gualou Qumai pill and Taohe Chengqi decoction were added and removed for 8 weeks, at one dose a day, and $400 \mathrm{~mL}$ of extract was extracted by water decoction and administered twice, in the morning and evening, after a meal. The results showed that there are 27 columns (90\%) were effective in the 30-column Gualou Qumai pill treatment group, indicating that the Gualou Qumai pill and Taohe Chengqi decoction can alleviate the symptoms of chronic prostatitis [3].

In a previous study, 60 patients with diabetic nephropathy, aged 40-75 years, with a duration of diabetes ranging from 4 to 10 years, were treated with a TCM decoction of modified Gualou Qumai (decocted in water twice) for 1 month, at one dose a day. Three hundred milliliters of warm decoction was administered twice, in the morning and evening. The results showed that there are 25 columns (83.33\%) were effective among the 30 columns of treatment groups, indicating that the Jiawei Gualou Qumai decoction can effectively improve the clinical symptoms of patients and assist in alleviating albuminuria and renal mesangial lesions, and delaying the process of diabetic nephropathy.

Dianthi herba is also effective in the treatment of urinary retention after an anorectal operation [85]. Recently, 60 patients with urinary retention after anorectal disease were selected and were administered Qin Yanning granules (20 g each time). The patients' first micturition time and voiding volume, inter alia, were measured. There are
55 (91.7\%) patients was effective Among the 60 patients, indicating that Qin Yanning granules could significantly improve the micturition and abdominal distension symptoms of patients and improve the clinical effect [86]. Shoulin used Gualou Qumai pill to treat patients with edema, and the reexamination showed that the total protein and albumin level, 24-h urine volume, and liver function were substantially improved, indicating that the Gualou Qumai pill can effectively improve the clinical symptoms of the patients [87]. The network diagram of chemical composition, pharmacological action and traditional application of Dianthi herba is shown in Fig. 13.

\section{Conclusion and perspective}

The traditional use, chemical composition, and extensive pharmacological activities of Dianthi herba are summarized based on information obtained from traditional literature records and modern literature. Dianthi herba has a long history of medicinal use in China. After decades of modern research, the main components such as saponins, flavonoids, peptides, anthraquinones and volatile oil have been isolated and identified, and their pharmacological activities have also been verified. Furthermore, through pharmacological experiments, the traditional applications of Dianthi herba have been confirmed, including the treatment of urinary tract infection and dysmenorrhea. However, more in-depth studies on the complex pharmacological effects of the Dianthus spp. and a complete phytochemical overview are needed to clarify the mechanism of action of Dianthi herba.

In modern times, $D$. superbus var. longicalycinus, $D$. versicolor, $D$. amtifolia, $D$. chinensis var. liaotungensis, and $D$. shandongensis are used as main sources of various medicines that can be confused with each other, and this could seriously affect treatment outcomes. According to the Chinese Pharmacopoeia, the identification methods for Dianthi herba are limited to thin layer chromatography and microscopy techniques. Therefore, it is necessary to establish an efficient, accurate, and scientific identification method to ensure the authenticity of the product. In addition, Dianthi herba medicinal materials are mostly found in wild medicinal materials. To ensure the conservation of sources of Dianthi herba medicinal materials and reduce the confusion of products and substitutes, attention should be paid to develop large-scale cultivation techniques. Studies should also focus on the establishment of a unified standard system and quality grade standards.

Saponins and cyclic peptides are considered the main pharmacologically active components in several bioactive compounds identified in Dianthi herba, and also new compounds isolated from Dianthi herba. Basic research 


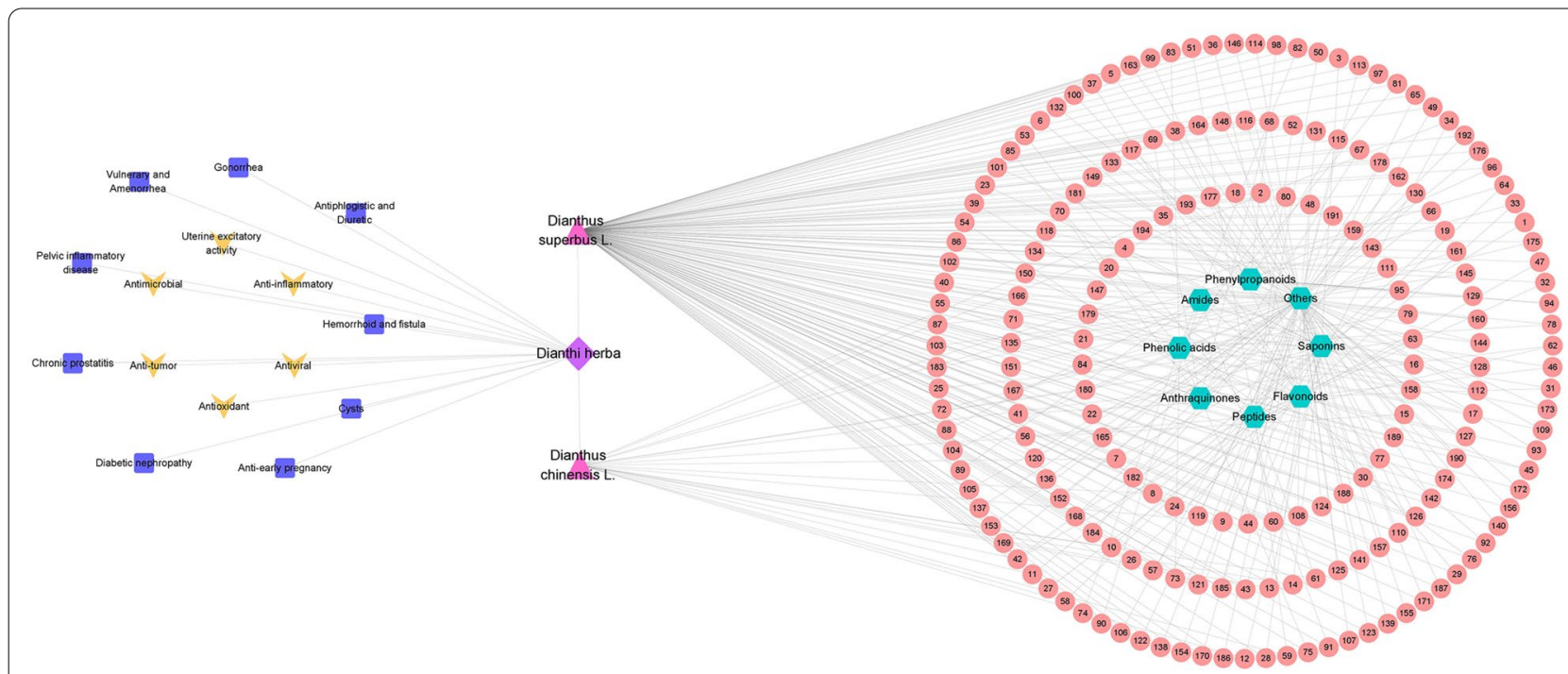

Fig. 13 Sources, chemical constituents, pharmacological effects and traditional applications of Dianthi herba

on the pharmacological activities of Dianthi herba is limited, focusing mainly on the activity of the extracted parts. Therefore, the study on the biological activity of other chemical components and the interaction and structureactivity relationship between saponins and cyclic peptides should be strengthened in the future. In addition, clinical studies should be conducted to effectively evaluate the efficacy, adverse reactions, and toxicity of Dianthi herba.

Currently, Dianthi herba is mainly composed of aboveground parts, and little is known about the composition and activity of the underground parts. Therefore, in-depth studies on other parts of the plant should be carried out in order to reveal its overall development potential. Finally, in addition to high efficacy and less adverse effects, a qualified drug should have good pharmacokinetic properties, but there are only a few studies on the pharmacokinetics of Dianthi herba, which greatly limits the development of the plant in the future market of traditional Chinese medicine.

Herein, we summarized the traditional applications, chemical composition, and pharmacological action of Dianthi herba globally. Further exploration is still needed to guarantee the rational utilization of Dianthi herba. Moreover, considerable attention should be paid to market demand and quality control.

\section{Abbreviations}

TCM: Traditional Chinese Medicine; PRC: People's Republic of China; CNKI: China National Knowledge Infrastructure; GBIF: Global Biodiversity Information Facility; GC-MS: Gas chromatography-mass spectrometry; NMR: Nuclear magnetic resonance; MTT: (3-(4,5-Dimethyl-2-Thiazolyl)-2,5-Diphenyl Tetrazolium
Bromide); MTS: 3-(4,5-Dimethylthiazol-2-yl)-5-(3-carboxymethoxyphenyl)-2-(4sulfophenyl)-2H-tetrazolium; DAPI: 4',6-Diamidino-2-phenylindole; RT-PCR: Reverse-Transcription polymerase chain reaction; Nrf2: Nuclear factor erythroid-2 related factor 2; ARE: Antioxidant response element; NQO1: NAD(P)H: quinone oxidoreductase 1; EtOAc: Ethyl acetate; ROS: Reactive oxygen species; Q3G: Quercetin 3-glucoside; $\mathrm{CC}_{50}$ : The half-maximal cytotoxic concentration; NA: Neuraminidase; GTP: Guanosine triphosphate; Q7G: Quercetin-7-Oglucoside; OVA: Ovalbumin; iNOS: Inducible nitric oxide synthase; BALF: Bronchoalveolar lavage fluid; IFNC: Interferon c; TGFB: Transforming growth factor beta; MIC: Minimum inhibitory concentration; MBC: Minimum bactericidal concentration; CRP: C-reactive protein.

\section{Supplementary Information}

The online version contains supplementary material available at https://doi. org/10.1186/s13020-022-00570-2.

Additional file 1. Distribution information of Dianthi herba.

\section{Acknowledgements}

Not applicable.

\section{Authors' contributions}

$\mathrm{QL}$ and $\mathrm{E}-\mathrm{HZ}$ drafted the manuscript. C-CW and Y-CL completed the figures and tables. $\mathrm{HN}$ collected literature. $\mathrm{YG}$ and $\mathrm{M}-\mathrm{HL}$ proposed the conception and designed the structure of the manuscript. All authors read and approved the final manuscript.

\section{Funding}

This work was supported by National Natural Science Foundation of China (Grant No. 81874336), 2019 Chinese medicine public health service subsidy special "the fourth survey on Chinese materia medica resource" (Grant No. Finance Society [2019] 39), China Agriculture Research System (Grant No. (ARS-21).

\section{Availability of data and materials}

All data generated or analysed during this study are included in this published article [and its additional files]. 


\section{Declarations}

Ethics approval and consent to participate

Not applicable.

\section{Consent for publication}

The manuscript is approved by all authors for publication.

\section{Competing interests}

The authors declare that they have no competing interests.

\section{Author details}

'Department of Pharmacy, Baotou Medical College, Baotou 014040, Inner Mongolia, China. ${ }^{2}$ Pharmaceutical Laboratory, Inner Mongolia Institute of Traditional Chinese Medicine, Hohhot 010020, Inner Mongolia, China. ${ }^{3}$ Inner Mongolia Key Laboratory of Characteristic Geoherbs Resources Protection and Utilization, Baotou Medical College, Baotou 014040, Inner Mongolia, China. ${ }^{4}$ Inner Mongolia Hospital of Traditional Chinese Medicine, Hohhot 010020, Inner Mongolia, China.

Received: 6 October 2021 Accepted: 7 January 2022

Published online: 21 January 2022

\section{References}

1. Cheng XM. Li Chuntang's experience in treating hydatoncus diseases with Dianthi herba as the main treatment. J Tradit Chin Med. 1999;8:505.

2. Wang HL, Jia CH, Zhan ZH, Pang ZR, Liu BS. Study on the law of drug compatibility in the treatment of drenching syndrome with traditional Chinese Medicine. Chin J Bas Med Tradit Chin Med. 2005;11(1):36-9.

3. Wang CG, Hu ZQ. Clinical observation on 30 cases of chronic prostatitis treated by Gualou Qumai pill combined with hetao chengqi decoction. Forum Tradit Chin Med. 2020;1:9-10.

4. Xiao H, Zhu XH, Ji MY, Yi LT, Li MH. Application of "Basaga" in mongolian medicine compound preparations in inner mongolia. Chin J Exp Tradit Med Form. 2020;26(3):190-8.

5. Liu C. Overview of pharmacological research on Dianthus superbus L. J Anhui Agric Sci. 2011;12(6):458-63.

6. Zhao YS, Bi YQ, Lei LJ, Zhu XH, Li MH. Variety systematization and research progress of mongolian medicine "bashaga." Chin J Chin Mater Med. 2017:42(5):998-1004

7. Zhang TJ, Xia GC. Pharmacognostical study on the Chinese drug Qumai. Chin Tradit Herbal Drugs. 1992;23(7):373-5.

8. Zhou FQ, Li JX, Zhang ZR, Wang ZF. Herbological studies of chinese drug "Qumai". Chin Med Mat. 1995;11:581-3.

9. Liu ZQ, Liu J. Identification of Dianthus superbus L. and confused varieties. Cap Food Med. 2016;23(3):63.

10. Zhang S, Yang CB. Optimization of microwave-assisted extraction of total saponins from Shaanxi Dianthus superbus L. by response surface methodology. J Shaanxi Agric Sci. 2018;64(5):16-20.

11. Chen X, Luo JG, Kong LY. Two new triterpenoid saponins from Dianthus superbus L. Asian Nat Prod Res. 2010;12(6):458-63.

12. Luo JG, Chen X, Kong LY. Three new triterpenoid saponins from Dianthus superbus. Chem Pharm Bull. 2011;59(4):518-21.

13. Ren $Y, X u X$, Zhang Q, Lu Y, Tian J. Isolation, characterization, and in rats plasma pharmacokinetic study of a new triterpenoid saponin from Dianthus superbus. Arch Pharm Res. 2014:40:2.

14. Tang XL, Yang XY, Jung HJ, Kim SY, Jung SY, Choi DY, Park WC, Park $\mathrm{H}$. Asiatic acid induces colon cancer cell growth inhibition and apoptosis through mitochondrial death cascade. Biol Pharm Bull. 2009:32(8):1399-405.

15. Li HY, Koike K, Ohmoto T, Ikeda K. Dianchinenosides A and B, two new saponins from Dianthus chinensis. Nat Prod. 1993;56(7):1065-70.

16. Ren-wei G, Rui-qi G, Hui-bin L, Jian-qiang L. Herbal textual research on Dianthi Herba. Mod Chin Med. 2020;22(11):158-65.

17. Li HY, Koike K, Ohmoto T, Li YS, Guo GF. L-dianose, a new monosaccharide from Dianthus chinensis L. Carbohyd Res. 1994;252:303-7.
18. Fu XY Tian JM Chemical constituents from Dianthus superbus. Chin Tradit Herbal Drugs. 2015;46(5):645-8.

19. Yang Y, Zhang W, Xie Z, Lei JC, Yu JP. Constituents of Dianthus superbus, and their cytotoxic activity. Chem Nat Compd. 2017;53(4):740-1.

20. Masumoto M, Okajima S. Two new species of pezothrips (thysanoptera, thripidae) in Japan and Europe, with designation of lectotype of type species. Zootaxa. 2020:4743(1):75-91.

21. Hong YJ, Xiao XF, Chen B, Yao SZ. Screening of angiotensin-converting enzyme inhibitors in traditional Chinese medicine by HPLC. Nat Prod Res Dev. 2008;20(4):728-30.

22. Nile SH, Kim DH, Nile A, Park GS, Gansukh E, Kai G. Probing the effect of quercetin 3-glucoside from Dianthus superbus L. against influenza virus infection- In vitro and in silico biochemical and toxicological screening. Food Chem Toxicol. 2019:35:110985.

23. Seraya LM, Birke K, Khimenko SV, Boguslavskaya LI. Flavonoid compounds of Dianthus superbus. Chem Nat Compd. 1978;14(6):684-5.

24. Li HY, Koike K, Ohmoto T. Triterpenoid saponins from Dianthus chinensis. Phytochemistry. 1994:35(3):751-6.

25. Sreelekshmi R, Siril EA, Muthukrishnan S. Role of biogenic silver nanoparticles on hyperhydricity reversion in Dianthus chinensis $L$, an in vitro model. Cul J Plant Grow Reg. 2021;1-17.

26. Ding C, Zhang W, Li J, Lei J, Yu J. Cytotoxic constituents of ethyl acetate fraction from Dianthus superbus. Nat Prod Lett. 2013;27(18):1691-4.

27. Gansukh E, Kazibwe Z, Pandurangan M, Judy G, Kim DH. Probing the impact of quercetin-7-O-glucoside on influenza virus replication influence. Phytomedicine. 2016;23(9):958-67.

28. Yun BR, Yang HJ, Weon JB, Lee J, Ma CJ. Simultaneous determination of eight bioactive compounds in Dianthus superbus by high-performance liquid chromatography. Pharmacogn Mag. 2016:12:264-S269.

29. Broderick SR, Evans WB. Biosolids promote similar plant growth and quality responses as conventional and slow-release fertilizers. HortTechnology. 2017;27(6):794-804.

30. Kim DH, Park GS, Nile AS, Kwon YD, Enkhtaivan G, Nile SH. Utilization of Dianthus superbus $L$ and its bioactive compounds for antioxidant, anti-influenza and toxicological effects. Food Chem Toxicol. 2019:125:313-21.

31. Zhu M, Duan X, Guo H, Huang W, Quan K, Yan X, Ji J, Li Y, Li Z. Occurrence of powdery mildew caused by Erysiphe buhrii on Dianthus chinensis in Inner Mongolia, China. Plant Dis. 2021. https://doi.org/10. 1094/PDIS-01-21-0048-PDN

32. Xu WY, Zhao SM, Zeng GZ, He WJ, Tan NH. Progress in the study of some important natural bioactive cyclopeptides. Acta Pharm Sin. 2012:47(3):271-9.

33. Wang YC, Tan NH, Zhou J, Wu HM. Cyclopeptides from Dianthus superbus. Phytochemistry. 1998;1998(49):1453-6.

34. Hsieh PW, Chang FR, Wu CC, Wu KY, Wu YC. New cytotoxic cyclic peptides and dianthramide from Dianthus superbus. Nat Prod. 2004;67(9):1522-7.

35. Tong Y, Luo JG, Wang R, Wang XB, Kong LY. New cyclic peptides with osteoblastic proliferative activity from Dianthus superbus. Bioorganicg Med Chem Lett. 2012;22:1908-11.

36. Han J, Wang Z, Zhang YQ, Zeng GZ, He WJ, Tan NH. New dicyclopeptides from Dianthus chinensis. Acta Pharm Sin. 2014:49(5):656.

37. Zhang S, Amso Z, De LRLM, Kaur H, Brimble MA. Synthesis of natural cyclopentapeptides isolated from Dianthus chinensis. J Nat Prod. 2016. https://doi.org/10.1021/acs.jnatprod.6b00152.

38. Wang XH, Chao QR, Huang H, Huang H, Wang T. Studies on the chemical constituents of fringed pink (Dianthus superbus). Chin Tradit Herb Drugs. 2000;4:10-1.

39. Guil-Guerrero JL, López-Martínez JC, Navarro-Juárez R, García-Maroto F, Campra-Madrid P. $\gamma$-linolenic acid from caryophyllaceae seed oil. J Am Oil Chem Soc. 2004:81(7):659-61.

40. Li G, Wen LM, Hou SZ, Cai DK, Li WX, Su ZR. RP-HPLC determination of emodin and chrysophanol contents in Ba zheng granules. Chin Tradit Herb Drugs. 2007;29(9):F11-3.

41. Fukai S, Nontaswatsri C, Uchiyama K. Agrobacterium-mediated genetic transformation of Dianthus hybrid'Telstar Scarlet'by using regenerable callus. Technical Bulletin of Faculty of Agriculture-Kagawa University (Japan). 2006 
42. Yun BR, Yang HJ, Weon JB, Lee J, Ma CJ. Neuroprotective properties of compounds extracted from Dianthus superbus L. against glutamateinduced cell death in HT22 cells. Pharmacogn Mag. 2016;12(46):109.

43. Yu JQ, Liao ZX, Cai XQ, Zou GL. Analysis of essential oil chemical compositions of Dianthus superbus with GC-MS. Chin Hosp Pharm J. 2008;28(2):157-8.

44. Li SQ, Zhang FL, Li J, Liu YW, Yu JQ. Screening and analysis of anti-tumor active components of petroleum ether extract from Dianthus superbus. Med J Wuhan Univ. 2014:35(1):11-5.

45. Han J, Huang M, Wang Z, Zheng Y, Zeng G, He W, Tan NH. Cyclopentapeptides from Dianthus chinensis. J Pept Sci. 2015;21(7):550-3.

46. Un-Kyo S, Ji-Cheon J. A study on antimutagenic activities of the extracts from dianthi herba. Herb Formula Sci. 2003:11:2.

47. Zhang S, Liu Z, Gao J, Wen J, Medicine SO. Study on optimization of total flavonoids of herba dianthi from Shaanxi in the extraction process by response surface method. World Chin Med. 2018;13(6):1541-5.

48. Cho HJ, Lee SJ, Kim S, Kim BD. Isolation and characterization of cdnas encoding ribosome inactivating protein from Dianthus sinensis L. Mol Cells. 2012;10(2):135-41.

49. Oh YC, Jeong YH, Cho WK, Hwang YH, Ma JY. Inhibitory effects of dianthi herba ethanolic extract on inflammatory and nociceptive responses in murine macrophages and mouse models of abdominal writhing and ear edema. J Ethnopharmacol. 2017. https://doi.org/10.1016/j.jep.2017. 09.010.

50. Kim KM, Lee H, Kang HJ, Lee YH, Yim YK. Anti-inflammatory effect of dianthi semen herbal-acupuncture at ki10 on nephritis in rats. $\mathrm{Kr} J$ Acupunct. 2009:26:1.

51. Horie T, Masuda K, Okada M, Takahashi K. Composition comprising an aqueous extract of red vine leaves and a diuretic. US. 2005;US20050142236A1.

52. Huh K, Ryu HM, Lee SI, Park JM, Song JW, Shin US. Studies on the uterotonic action of $N$-butanol extracts from dianthi herba. J Soc Pharmacogn. 1988;19(4):256-61.

53. Lee JM, Lee $\mathrm{CH}$, Cho JH, Jang JB, Lee KS, Kim ES. Antimicrobial effects of herbs for removing dampness and promoting urination against vaginal microbe. J Kr Obstet Gynecol. 2007;20(1):001-15.

54. Lei S, Jiang YY, Liu B. Oligopeptides in plant medicines cited in chinese pharmacopoeia. China J Chin Mater Med. 2016;41(16):2943.

55. Zhang JY, Yi T, Liu J, Zhao ZZ, Chen HB. Quercetin induces apoptosis via the mitochondrial pathway in KB and KBv200 cells. J Agr Food Chem. 2013;61(9):2188-95.

56. Nho KJ, Chun JM, Kim HK. Ethanol extract of Dianthus chinensis L. induces apoptosis in human hepatocellular carcinoma HEPG2 cells in vitro. Evid Based Complement Alternat Med. 2012;1:573527.

57. Shin JA, Kim JJ, Choi ES, Shim JH, Ryu MH, Kwon KH, Park HM, Seo JY, Lee SY, Lim DW, Cho NP, Cho SD. In vitro apoptotic effects of methanol extracts of Dianthus chinensis and Acalypha australis L. targeting specificity protein 1 in human oral cancer cells. Head Neck. 2013;35(7):992-8

58. Yan CL, An FY, Liu YQ, Wang JL, Liu XS, Zhao WK, Yang XR, Pan T. Mechanism exploration of Qumai decoction on the oxidative stress damage in calcium oxalate stone rats via nrf2/are signal pathway. Chin J Mod Appl Pharm. 2019;36(17):11-5.

59. Yu JQ, Yin Y, Lei JC, Zhang XQ, Chen W, Ding CL. Activation of apoptosis by ethyl acetate fraction of ethanol extract of Dianthus superbus in HepG2 cell line. Cancer Epidemiol. 2012;36(1):e40-5.

60. Gao W, Guo DH, Zhang XZ, Guo YX. Study on extraction of flavonoids from Dianthus superbus assisted with Aspergillus niger fermentation and its antioxidant activity. J Anhui Agric Sci. 2017;45(32):142-3.

61. Khan SR. Nephrocalcinosis in animal models with and without stones. Urol Res. 2010;38(6):429-38

62. Yu JO, Liao ZX, Lei JC, Hu XM. Antioxidant and cytotoxic activities of various fractions of ethanol extract of Dianthus superbus. Food Chem. 2007;104(3):1215-9.

63. Stefaniak A, Grzeszczuk ME. Nutritional and biological value of five edible flower species. Not Bot Horti Agrobot Cluj-Napoca. 2018;47:1.

64. Gao H, Xu P, Li J, Ji H, Xia X. AgNO3 prevents the occurrence of hyperhydricity in Dianthus chinensis L. by enhancing water loss and antioxidant capacity. In Vitro Cell Dev Biol Plant. 2017;53(6):561-70.

65. Shin IS, Lee MY, Ha H, Jeon WY, Seo CS, Shin HK. Dianthus superbus fructus suppresses airway inflammation by downregulating of inducible nitric oxide synthase in an ovalbumin-induced murine model of asthma. J Inflamm. 2012;9(1):41-41.

66. Miao MS, Cheng BL, Jiang N, Jiang MQ. Qumai total flavonoids uced diabetic mice model of streptozotocin. Appl Mech Mater. 2014:664:410-4.

67. Reid-Adam J, Yang N, Song Y, Cravedi P, Li XM. Immunosuppressive effects of the traditional chinese herb Qumai on human alloreactive T cells. Am J Transplant. 2013;13(5):1159-67.

68. Slotkin W, Exposito IL, Castillo A, Mishoe M, Yang N, Liang BH, Li XM. Compounds isolated from Qumai (Dianthus superbus) inhibit ige secretion by human b cells. J allergy Clin Immun. 2010;125(2):120-120.

69. Jia $S$, Jhy $B$, Jls $B$, Jiang CS, Zhang $H$. Two new quinolone alkaloids from Dianthus superbus var. superbus. Tetrahedron Lett. 2019;60(2):161-3.

70. Xu XD, Wei YP. Clinical analysis report of treatment of ureteral calcul with sijin Qumai decoction combined with extracorporeal shockwave lithotripsy. Med frontier. 2015;5(31):200-1.

71. Li DG, Zhou FQ, Ji GC, Zhang ZM, Shi RH. Study on the natriuresis of herba dianthi growing in shandong province. J Chin Med Mater. 1996;19(10):520-2.

72. Deng JD, Shen L. Diuretic tonglin tiaoxue decoction for the treatment of chronic urinary tract infection randomized parallel control study. J Pract Tradit Chin Intern Med. 2015;10:45-6.

73. Yoon JJ, Park JH, Kim HJ, Jin HG, Kim HY, Ahn YM, Kim YC, Lee HS, Lee YJ, Kang DG. Dianthus superbus improves glomerular fibrosis and renal dysfunction in diabetic nephropathy model. Nutrients. 2019;11(3):553.

74. Yang XY, Li C, Ma SY, Li JH. Effects of the additives and the combination of plants on pb absorption, growth and quality of Dianthus superbus. Chin J Appl Eco. 2017;28:1155-60.

75. Li XG, Wang JY, Liu Y, Lin Y, Zeng SH. Experimental study on the antiimplantation of Diathus superbus L. fruit extract. Pharm Clin Chin Mater Med. 2012;2(5):24-6.

76. Li XG, Gao XM. Experimental study of the effects of Dianthus superbus decoction on mouse pregnancy. J Beijing Univ Tradit Chin Med. 2000;23(6):40.

77. Guo LF, Wen FH, Li XM, Jiao JJ, Song HY. Excitatory effect of Dianthus superbus $L$. on rat uterus in vitro and rabbit uterus in vivo and its synergistic effect with prostaglandin E2. Tianjin Pharm. 1983;5:268-71.

78. Li JJ, Tu YY, Tong JZ, Wang PT. Inhibitory activity of Diathus superbus L. and 11 kinds of diuretic traditional chinese medicines for urogenital chlamydia trachomatis in vitro. Chin J Tradit Chin Med. 2000;10:52-4

79. Dahiya R. Synthesis and biological activity of a cyclic hexapeptide from Dianthus superbus. Chem Pap. 2008:62(5):527-35.

80. Blavet N, Charif D, Oger-Desfeux C, Marais GA, Widmer A. Comparative high-throughput transcriptome sequencing and development of SiESTa, the silene EST annotation database. BMC Genomics. 2011;26(12):376.

81. Yoon J, Park J, Kim H, Jin HG, Kim H, Ahn YM. Dianthus superbus. improves glomerular fibrosis and renal dysfunction in diabetic nephropathy model. Nutrients. 2019;11:3.

82. Zhang HY, Zhu H, Sun F, Hui XD, Zhao B. The effect of Gualouqumai decoction on CRP, IL- 6 and TGF- $\beta 1$ in renal tissue of diabetic nephropathy rats. Jiangsu J Tradit Chin Med. 2016;48(7):77-9.

83. Iván LE, Castillo A, Yang N, Liang B, Li XM. Chinese herbal extracts of Rubia cordifolia and Dianthus superbus suppress IgE production and prevent peanut-induced anaphylaxis. Chin Med. 2011;6(1):35.

84. Choi HS, Hong JS, Geronimo FK, Kim LH. Implications of CaCl2 application to plants in LID facilities. Water Sci Technol. 2018;78(5):1045-53.

85. Lu X, Zheng LQ, Hu MY. Clinical study on modified gualou Qumai decoction combined with conventional western medicine in treating 30 cases of diabetic nephropathy at stage III-IV. Jiangsu J Tradit Chin Med. 2019;51(8):39-41.

86. Huang Y, Liu CW, Zhi YX. Effect of mi yanning granules on urinary retention after operation of anorectal diseases. J Guangxi Univer Chin Med. 2020:3:32-4.

87. Ding N, Cui W, Zhang SL. Application of gualou Qumai pill in the treatment of edema cardiovascular disease electronic. J Integr Tradit Chin West Med. 2019;1:9-10.

88. Pei GL, Huang HQ, Sun QG, Liang CM, Tian Y. Qumai tea for cysts. China's Naturopath. 2016;12:61-2 
89. Yan YL. Diabetic nephropathy and the classical prescription gualou Qumai pill. Henan Tradit Chin Medicine. 2007:4:11-2.

90. Liu J, Zhang RY. Gualou Qumai decoction in treating 66 cases of chronic prostatitis". J Pract Tradit Chin Intern Med. 2005;8:468.

91. Ma X, Zhang SR. Treatment of 60 cases of pelvic inflammatory mass with Qumai decoction. Shanxi J Tradit Chin Med. 2002;1:73.

92. Zhu XL. Gualou Qumai pills for treatment of elderly prostatic hypertrophy. J Sichuan Tradit Chin Med. 1992;4:35.

93. Slotkin W, Exposito IL, Castillo A, Mishoe M, Yang N, Liang BH, Li XM. Compounds isolated from Qu Mai (Dianthus superbus) inhibit IgE secretion by human B cells. J Allergy Clin Immun. 2010;125(2):S81-94.

94. Wang YT, Guo YC, Sun J. Treatment of 2 cases of diabetes mellitus complicated with nephropathy high edema with modified Gualouqumai Pill [D]. Jilin J Tradit Chin Med. 2001;21(6):58.

95. Szego A, Lukacs N, Toth EK, Potyondi L. Long term survival of cryptic viruses in aseptically grown in vitro propagated plants. Acta Hortic. 2006;505-510.

96. Zhang S, Hong HU, Wang Y, Wang F. Effect of ethanol and water extract from Dianthus superbus on antimicrobial infection in mice. Chin Archi Tradit Chin Med. 1807:2018(7):1771-3.

97. Ademosun AO, Oboh G, Passamonti S, Tramer F, Ziberna L, Boligon AA, Athayde ML. Phenolics from grapefruit peels inhibit HMG-CoA reductase and angiotensin-I converting enzyme and show antioxidative properties in endothelial EA.Hy 926 cells. Food Sci Hum Well. 2015:4(2):80-5.

98. Irondi EA, Adegoke BM, Effion ES, Oyewo SO, Alamu EO, Boligon AA. Enzymes inhibitory property, antioxidant activity and phenolics profile of raw and roasted red sorghum grains in vitro. Food Sci Hum Well. 2019:8(2):142-8

99. El-Toumy SA, Salib JY, El-Kashak WA, Marty C, Bedoux G, Bourgougnon N. Antiviral effect of polyphenol rich plant extracts on herpes simplex virus type 1. Food Sci Hum Well. 2018;7(1):91-101.

100. Rajendiran V, Natarajan V, Devaraj SN. Anti-inflammatory activity of Alpinia officinarum hance on rat colon inflammation and tissue damage in DSS induced acute and chronic colitis models. Food Sci Hum Well. 2018:7(4):273-81.

101. Sachin V, Li BS, Vishwaraman M, Prasad T. Anti-inflammatory and anti-arthritic activity of type-A procyanidine polyphenols from bark of Cinnamomum zeylanicum in rats. Food Sci Hum Well. 2013;2(2):59-67.

102. Li XG, Gao XM. Experimental study on the effect of Qumai decoction on pregnancy in mice[J]. J Beijing Univ Tradit Chin Med. 2000;06:40-2.

103. Zhang N, Zhang ZY, Wang XM, Chi BF, Su ZG, Cao XD. Study on the toxic effect of Mongolian medicine Qianmai on mice. Chin Med Pharm. 2021;11(01):33-7.

104. Hsieh PW, Chang FR, Wu CC, Li CM, Wu KY, Chen SL, Yen HF, Wu YC. Longicalycinin A, a new cytotoxic cyclic peptide from Dianthus superbus var. longicalycinus (MAXIM.) WILL. Chem Pharm Bull. 2005;53(3):336-8.

105. Yu JQ, Yin Y, Lei JC, Zhang XQ, Chen W, Ding CL, Wu S, He XY, Liu YW, Zou GL. Activation of apoptosis by ethyl acetate fraction of ethanol extract of Dianthus superbus in HepG2 cell line. Cancer Epidemiol. 2012:36(1):e40-5.

106. Zhang $\mathrm{S}, \mathrm{Hu} \mathrm{H}$, Wang $\mathrm{Y}$. Study on the effect of ethanol and water extract of wheat produced in Shaanxi on antibacterial infection in mice. Chin Arc Trad Chin Med. 2018;36(7):1771-3.

107. Dong XC, Fu W. Fourteen methods for internal treatment of hemorrhoids and fistulas[J]. Yunnan J Tradit Chin Med Mater Medica. 1998;04:20-1.

108. Durairaj A, Gandhiraman K. Complications and management of paraovarian cyst: a retrospective analysis. J Obstet Gynaecol India. 2019:69(2):180-4

109. Casadei L, Dominici F, Scaldaferri D, Vicomandi V, Ciacci S, Piccione E. Anti-Müllerian hormone levels and spontaneous pregnancy in women undergoing surgery for benign ovarian cysts. Gynecol Endocrinol. 2018:34(10):909-12

110. Yang $\mathrm{H}$, Yin J. Clinical efficacy of traditional Chinese Medicine in the treatment of Ovarian cyst. Jilin J Tradit Chin Medical. 2020;40(04):474-6.

111. Baek IJ, Yon JM, Lee SR, Yoo WJ, Nahm SS, Kim YB, Nam SY. Effects of Banha (Pinellia ternata) on the gene expression of GPx family in the reproductive systems of male rats. Lab Anim Res. 2006;22(1):97-103.
112. Song $X Q$, Wei $H P$, Li DD, Liao QL, Chen CH, An M. Experimental study on the effect of Carthami Flos on early pregnancy in mice. Chin Tradit Pat Med. 2014:36(11):2408-10.

113. Li L. Observation on the effect of Arnebiae Radix on Drug abortion Hemorrhage. Guizhou Med J H. 2004;28(7):629.

114. Zhang XC, Wang M. Research progress on pharmacological effects and clinical application of traditional Chinese Medicine in the treatment of diabetic nephropathy. Chin J Exp Tradit Med Form. 2019;25(09):228-34.

115. Li H, Hung A, Yang AWH. A classic herbal formula Danggui Beimu Kushen Wan for chronic prostatitis: from traditional knowledge to scientific exploration. Evid Based Complement Alternat Med. 2018:2018:1612948

116. Bae JS, Park HS, Park JW, Li SH, Chun YS. Red ginseng and 20(S)-Rg3 control testosterone-induced prostate hyperplasia by deregulating androgen receptor signaling. J Nat Med. 2012;66(3):476-85.

117. Li LF, Fu JF. Observation on the effect of traditional Chinese medicine retention enema on chronic pelvic inflammation and inflammatory response factors. World J Tradit Chin Med. 2017:12(04):772-5.

118. Yang HH, Tang JQ, Han T, Zhang YC, Haung HL. Analysis on the regularity of prescription use of traditional Chinese Medicine in the treatment of Secondary amenorrhea in recent 10 years. Shandong J Tradit Chin Med. 2017;36(02):140-2.

119. Song LL, Gao X. Clinical study of Xiehuo jiedu decoction in the treatment of chronic gonorrhea. Chin Her Med. 2009;6(15):84-6.

120. Chen $X Y$, Yang $Z H$, Hong MH. Analysis of quality Standard of diuretic mixture. Chin Med Pharm. 2020;10(16):245-8.

121. Hsu YL, Kuo PL, Lin LT, Lin CC. Asiatic acid, a triterpene, induces apoptosis and cell cycle arrest through activation of extracellular signalregulated kinase and p38 mitogen-activated protein kinase pathways in human breast cancer cells. J Pharmacol Exp Ther. 2005;313(1):333-44.

122. Park BC, Bosire KO, Lee ES, Lee YS, Kim JA. Asiatic acid induces apoptosis in SK-MEL-2 human melanoma cells. Cancer Lett. 2005;218(1):81-90.

123. Hao Y, Huang J, Ma Y, Chen W, Fan Q, Sun X, Shao M, Cai H. Asiatic acid inhibits proliferation, migration and induces apoptosis by regulating $\mathrm{Pdcd} 4$ via the PI3K/Akt/mTOR/p70S6K signaling pathway in human colon carcinoma cells. Oncol Lett. 2018;15(6):8223-30.

124. Kazakova OB, Giniyatullina GV, Yamansarov EY, Tolstikov GA. Betulin and ursolic acid synthetic derivatives as inhibitors of Papilloma virus. Bioorg Med Chem Lett. 2010;20(14):4088-90.

125. Nakagawa-Goto K, Yamada K, Taniguchi M, Tokuda H, Lee KH. Cancer preventive agents 9 . Betulinic acid derivatives as potent cancer chemopreventive agents. Bioorg Med Chem Lett. 2009;19(13):3378-81.

\section{Publisher's Note}

Springer Nature remains neutral with regard to jurisdictional claims in published maps and institutional affiliations.

\footnotetext{
Ready to submit your research? Choose BMC and benefit from:

- fast, convenient online submission

- thorough peer review by experienced researchers in your field

- rapid publication on acceptance

- support for research data, including large and complex data types

- gold Open Access which fosters wider collaboration and increased citations
}

- maximum visibility for your research: over 100M website views per year

At BMC, research is always in progress.

Learn more biomedcentral.com/submissions 